\title{
Squeeze Film Dampers Executing Small Amplitude Circular-Centered Orbits in High-Speed Turbomachinery
}

\author{
Sina Hamzehlouia and Kamran Behdinan \\ Department of Mechanical and Industrial Engineering, University of Toronto, Toronto, ON, Canada \\ Correspondence should be addressed to Sina Hamzehlouia; shamzehl@mie.utoronto.ca
}

Received 11 April 2016; Revised 3 November 2016; Accepted 16 November 2016

Academic Editor: Linda L. Vahala

Copyright ( $) 2016$ S. Hamzehlouia and K. Behdinan. This is an open access article distributed under the Creative Commons Attribution License, which permits unrestricted use, distribution, and reproduction in any medium, provided the original work is properly cited.

\begin{abstract}
This work represents a pressure distribution model for finite length squeeze film dampers (SFDs) executing small amplitude circular-centered orbits (CCOs) with application in high-speed turbomachinery design. The proposed pressure distribution model only accounts for unsteady (temporal) inertia terms, since based on order of magnitude analysis, for small amplitude motions of the journal center, the effect of convective inertia is negligible relative to unsteady (temporal) inertia. In this work, the continuity equation and the momentum transport equations for incompressible lubricants are reduced by assuming that the shapes of the fluid velocity profiles are not strongly influenced by the inertia forces, obtaining an extended form of Reynolds equation for the hydrodynamic pressure distribution that accounts for fluid inertia effects. Furthermore, a numerical procedure is represented to discretize the model equations by applying finite difference approximation (FDA) and to numerically determine the pressure distribution and fluid film reaction forces in SFDs with significant accuracy. Finally, the proposed model is incorporated into a simulation model and the results are compared against existing SFD models. Based on the simulation results, the pressure distribution and fluid film reaction forces are significantly influenced by fluid inertia effects even at small and moderate Reynolds numbers.
\end{abstract}

\section{Introduction}

According to the classic lubrication theory, the pressure distribution in the annular region of a squeeze film damper is determined by using Reynolds equation, where it is assumed that the inertial forces are negligible relative to viscous forces (i.e., $\mathrm{Re} \approx 0$ ) [1]. In recent years, increase in velocity and size of turbomachinery and application of low-viscosity lubricants requires the fluid inertia effect to be included in design and analysis of SFDs.

Generally, SFDs incorporate an oil film to provide load support with a suitable damping action. SFDs are typically implemented in rotor systems to attenuate vibration amplitudes and to isolate structural components in highspeed turbomachinery applications, including aircraft turbine engines, high performance compressors, and automotive turbochargers. The main advantages of SFDs are their capacity in reducing the effects of unbalance-induced rotor vibrations at resonance frequencies, reducing the level of forces transmitted at supports, and preventing nonsynchronous instabilities. Figure 1 demonstrates the geometry of a conventional SFD. A typical SFD consists of a stationary outer bearing and an inner journal with approximately identical diameters. The journal is assembled on the outer surface of a rolling element and is prevented from rotation by using an antirotation pin. The annular region between the bearing and the housing is generally smaller than $0.25 \mathrm{~mm}$ and is filled with a lubricant. The precession motion of the journal is induced by residual unbalance of the rotor and generates a hydrodynamic squeeze film pressure distribution that exerts reaction forces over the journal, providing the damping force to attenuate the transmitted forces and in turn reducing the rotor vibration. The dynamic force response of SFDs is determined by the damper geometry, operating speed, and lubricant properties. Furthermore, SFDs do not produce direct stiffness; a suitable degree of stiffness is introduced by using retaining springs parallel to the squeeze film or by using a pair of elastomer O-rings in radial disposition.

The pioneering work by Cooper [2] demonstrated the benefits conferred on the rotor operation from coupling of 


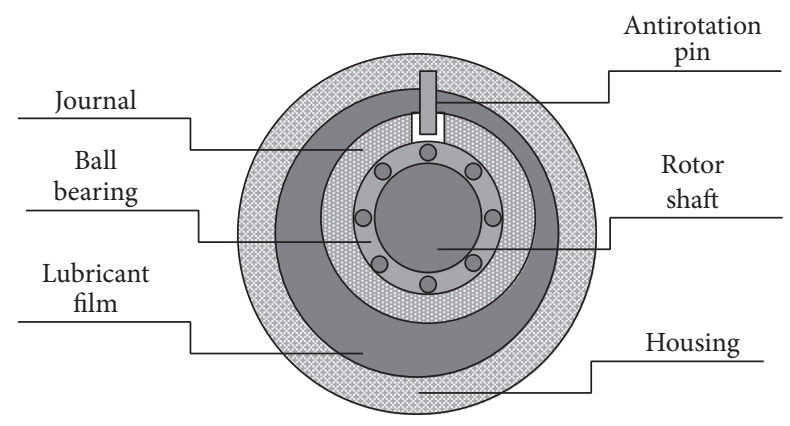

Figure 1: Schematic diagram of a squeeze film damper.

a damping element, represented by an oil squeeze film, to an elastic element placed within the rotor support. Ever since, research works have focused on providing greater insight into different features of SFDs, including the effect of fluid inertia. The effect of fluid inertia on the performance of SFDs has been the subject of several studies in the past few decades [3, 4]. Smith [5] used simplified journal bearing geometries, including the short and long bearing models, to determine the effect of fluid inertia on the dynamic characteristics of journal bearings. He concluded that the effect of fluid inertia introduces an added mass to the rotor system, which significantly influences the dynamic characteristics of short stiff rotors. Several investigations $[6,7]$ have used the limiting damper geometries (i.e., short bearing and long bearing approximations) to determine the effect of fluid inertia on bearing force characteristics. ElShafei and Crandall [8] have used an energy approximation method by applying Lagrange's equation to determine the force coefficients in SFDs. They have shown that the energy approximation provides an accurate estimation of the inertia forces in SFDs. Moreover, they have determined the force coefficients for long and short SFDs. San Andres et al. [912] have determined SFD force coefficients for short and long bearing geometries and extended the application of these force coefficients to finite length bearings by applying a leakage coefficient. Hashimoto [13] has developed analytical pressure expressions for infinitely long journal bearings with turbulence and inertia effects. El-Shafei [14-16] has determined the SFD force coefficients including inertia effects for both short and long dampers and incorporated the results into rotordynamic models. Although the analysis of limiting bearing geometries provides enhanced understanding of the flow dynamics for SFDs, they demonstrate boundary properties of the dampers, while, in practice, dampers have a finite length and application of the above approximations leads to significant calculation error for arbitrary finite length SFD geometries and operating conditions.

Reinhardt and Lund [17] introduced a first-order perturbation technique for small Reynolds numbers to determine the force coefficients for hydrodynamic journal bearings. They suggested that the inertial corrections to the damping and stiffness coefficients are small, while a large inertia coefficient is introduced that significantly affects the bearing dynamics. However, application of the first-order perturbation method is limited to small and moderate inertia effects.

An alternative approach is to introduce a bulk-flow model $[18,19]$ for the SFD. Subsequently, the bulk-flow model is solved by using finite element method (FEM). While the bulk-flow modeling and the numerical solution significantly improve the accuracy of the calculation for SFD parameters, it is generally computationally very inefficient, especially for integration into rotordynamic models, where the SFD parameters are calculated over several iterations.

Andrés and Delgado [20] have represented a modified Reynolds equation to describe the lubricant pressure distribution in SFDs and proposed a solution based on FEM. San Andrés and Vance [21] have proposed an analytical closedform expression for the lubricant pressure in SFDs including fluid inertia and turbulence.

Circular-centered motions of the journal center are a very common type of journal motion in industrial applications of squeeze film dampers, including vertical rotors mounted on SFDs, horizontal rotors mounted on SFDs with centralizing springs, and rotors operating close to critical velocities and for rotor response to large unbalance forces [9]. This CCO condition is typically assumed for SFDs with centering elements. Furthermore, the assumption of small amplitude motions of the journal center is a very common practice in the turbomachinery industry, which further reduces the SFD flow equations by eliminating the nonlinear convective inertia terms. This model reduction facilitates the investigation of unsteady inertia terms. Tichy [22] has studied the effect of small amplitude CCOs for viscoelastic fluids, showing the significant effect of fluid inertia on the fluid film reaction forces, even at moderate Reynolds numbers. San Andres and Vance $[9,11]$ have further emphasized the significant effect of fluid inertia on dynamic performance of squeeze film dampers executing small amplitude CCOs by representing the effect of fluid inertia on SFD force coefficients for both central and off-centered motions of the journal center.

In most studies, the dynamic characteristics of SFDs are investigated for an isolated bearing system and the models are constructed to satisfy computational requirements for a single SFD system; however, in practice, SFDs are integrated into a rotor system. The rotordynamic analysis of the rotor system, including the dynamic interaction between the SFDs and the rotor, requires the development of an SFD model that is designed for rotordynamic applications, including significant accuracy and maintaining an acceptable computational efficiency. The complexity of most current SFD dynamic models makes them computationally inefficient for rotordynamic analysis. Furthermore, the application of approximate SFD geometries (i.e., long and short bearing models) for the finite length bearings results in considerable degradation of the calculation accuracy.

This work introduces a dynamic model for the calculation of fluid film reaction forces for finite length squeeze film dampers executing small amplitude CCOs. The proposed model numerically determines the SFD fluid film reaction force components under different operating conditions. By 
using an efficient numerical technique, the direct calculation of SFD fluid forces makes the proposed model very ideal for rotordynamic applications. The resulting extended Reynolds equation is used to numerically determine the pressure distribution in the lubricant. Finally, the pressure distribution is numerically integrated over the journal surface to calculate the fluid film reaction force components.

\section{Governing Equations}

The dynamic behavior of a viscous Newtonian fluid within boundaries is generally characterized by using the 3dimensional Navier-Stokes equations as follows [1]:

$$
\begin{aligned}
& \frac{\partial \rho}{\partial t}+\frac{\partial(\rho u)}{\partial x}+\frac{\partial(\rho v)}{\partial y}+\frac{\partial(\rho w)}{\partial z}=0 \\
& \rho\left\{\frac{\partial u}{\partial t}+u \frac{\partial u}{\partial x}+v \frac{\partial u}{\partial y}+w \frac{\partial u}{\partial z}\right\} \\
& =-\frac{\partial P}{\partial x}+\mu\left\{\frac{\partial^{2} u}{\partial x^{2}}+\frac{\partial^{2} u}{\partial y^{2}}+\frac{\partial^{2} u}{\partial z^{2}}\right\}, \\
& \rho\left\{\frac{\partial v}{\partial t}+u \frac{\partial v}{\partial x}+v \frac{\partial v}{\partial y}+w \frac{\partial v}{\partial z}\right\} \\
& =-\frac{\partial P}{\partial y}+\mu\left\{\frac{\partial^{2} v}{\partial x^{2}}+\frac{\partial^{2} v}{\partial y^{2}}+\frac{\partial^{2} v}{\partial z^{2}}\right\}, \\
& \rho\left\{\frac{\partial w}{\partial t}+u \frac{\partial w}{\partial x}+v \frac{\partial w}{\partial y}+w \frac{\partial w}{\partial z}\right\} \\
& =-\frac{\partial P}{\partial z}+\mu\left\{\frac{\partial^{2} w}{\partial x^{2}}+\frac{\partial^{2} w}{\partial y^{2}}+\frac{\partial^{2} w}{\partial z^{2}}\right\},
\end{aligned}
$$

where (1) is the continuity equation corresponding to the conservation of mass within the fluid boundaries and (2) to (4) correspond to the conservation of momentum within the fluid boundaries.

According to the hydrodynamic lubrication theory, the thin film assumption in hydrodynamic bearings is characterized by the small ratio of film thickness to the bearing's other physical dimensions; that is, $c \ll R$. Consequently, we have the following:

(1) The effect of the curvature of the film is negligible; hence a linear coordinate system is used to describe the lubricant dynamics.

(2) The variation of the pressure across the film is negligible (i.e., $\partial P / \partial y=0$ ).

The SFD configuration in this work is a symmetric damper about its midplane with open ends (i.e., no seal). The

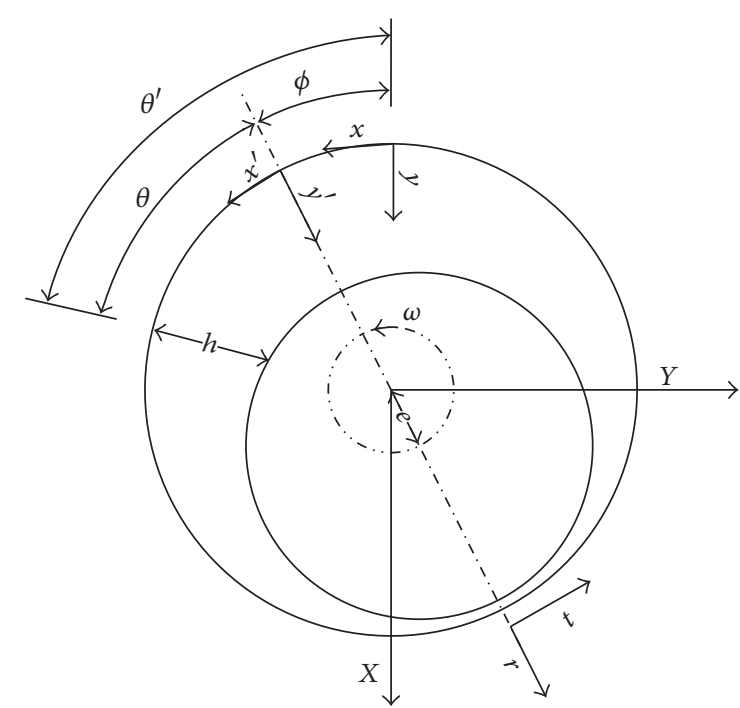

FIGURE 2: Squeeze film damper geometry and coordinate systems.

geometry of the system is represented in Figure 2. According to the thin film theory, which facilitates the application of Cartesian coordinates, an orthogonal Cartesian coordinate system $\{x, y, z\}$ is fixed in the plane of the lubricant, where the $z$-axis is perpendicular to the plane of motion. Furthermore, an orthogonal Cartesian system $\left\{x^{\prime}, y^{\prime}, z^{\prime}\right\}$ translating with angular velocity $R \omega$ is introduced, where the $x^{\prime}$-axis is perpendicular to the line connecting the centers of the inner and outer cylinders, and the $y^{\prime}$-axis is in the direction of the minimum thickness. The angle $\theta^{\prime}$ starts from the origin of the fixed Cartesian system and the angle $\theta$ is measured from the maximum film thickness in the direction of the whirling motion. Finally, a fixed inertial coordinate system $\{X, Y\}$ is defined at the center of the bearing. Based on the thin film theory, for an incompressible and isoviscous lubricant, the flow equations in the SFD are reduced to the following:

$$
\begin{gathered}
\frac{\partial u}{\partial x}+\frac{\partial v}{\partial y}+\frac{\partial w}{\partial z}=0 \\
\rho\left\{\frac{\partial u}{\partial t}+u \frac{\partial u}{\partial x}+v \frac{\partial u}{\partial y}+w \frac{\partial u}{\partial z}\right\}=-\frac{\partial P}{\partial x}+\mu \frac{\partial^{2} u}{\partial y^{2}}, \\
\frac{\partial P}{\partial y}=0 \\
\rho\left\{\frac{\partial w}{\partial t}+u \frac{\partial w}{\partial x}+v \frac{\partial w}{\partial y}+w \frac{\partial w}{\partial z}\right\}=-\frac{\partial P}{\partial z}+\mu \frac{\partial^{2} w}{\partial y^{2}} .
\end{gathered}
$$

Subsequently, the flow equations are normalized by means of dimensionless quantities to demonstrate the 
effect of Reynolds number on the flow characteristics:

$$
\begin{gathered}
\theta=\theta^{\prime}-\phi=\frac{x}{R}-\omega t, \eta=\frac{y}{c}, \xi=\frac{z}{R}, \tau=\omega t, \\
\bar{u}=\frac{u}{R \omega}, \bar{v}=\frac{v}{c \omega}, \bar{w}=\frac{w}{R \omega}, \\
\bar{P}=\frac{P c^{2}}{R^{2} \omega \mu}, H=\frac{h}{c}=1+\varepsilon \cos \theta, \operatorname{Re}=\frac{\rho \omega c^{2}}{\mu} .
\end{gathered}
$$

Furthermore, according to order of magnitude calculations, for small amplitude motions, convective inertia terms in (6) and (8) are negligible relative to unsteady (temporal) inertia terms [23]. Therefore, the dimensionless flow equations are introduced as follows:

$$
\begin{aligned}
\frac{\partial \bar{u}}{\partial \theta}+\frac{\partial \bar{v}}{\partial \eta}+\frac{\partial \bar{w}}{\partial \xi} & =0 \\
\operatorname{Re} \frac{\partial \bar{u}}{\partial \tau} & =-\frac{\partial \bar{P}}{\partial \theta}+\frac{\partial^{2} \bar{u}}{\partial \eta^{2}} \\
\operatorname{Re} \frac{\partial \bar{w}}{\partial \tau} & =-\frac{\partial \bar{P}}{\partial \xi}+\frac{\partial^{2} \bar{w}}{\partial \eta^{2}}
\end{aligned}
$$

The boundary conditions for the fluid velocities in SFDs are defined as follows:

$$
\begin{gathered}
\bar{u}=0, \quad \bar{v}=0, \bar{w}=0, \quad \eta=0, \\
\bar{u}=0, \bar{v}=\frac{\partial H}{\partial \tau}, \bar{w}=0, \quad \eta=H .
\end{gathered}
$$

Additionally, the boundary conditions for the hydrodynamic pressure in an open ended SFD are as follows.

(1) The pressure is periodic and continuous in the circumferential direction $(\theta)$; that is,

$$
\bar{P}(\theta, \xi)=\bar{P}(\theta+2 \pi, \xi) .
$$

(2) The lubricant pressure equals atmospheric pressure at the axial ends of the bearing; that is,

$$
\bar{P}\left(\theta, \frac{L}{D}\right)=\bar{P}\left(\theta,-\frac{L}{D}\right)=0 .
$$

(3) The hydrodynamic pressure must be above the liquid cavitation pressure; that is,

$$
\bar{P} \geq \bar{P}_{\text {cav }} \quad 0 \leq \theta \leq 2 \pi,-\frac{L}{D} \leq z \leq \frac{L}{D},
$$

where $P_{\text {cav }}$ is the saturation pressure of the lubricant or the saturation pressure for the release of entrapped gases in the lubricant, typically ambient pressure. Subsequently, (10) are integrated along the film thickness and the velocity boundary conditions are applied:

$$
\begin{aligned}
\frac{\partial}{\partial \theta} \int_{0}^{H} \bar{u} d \eta+\frac{\partial}{\partial \xi} \int_{0}^{H} \bar{w} d \eta+\frac{\partial H}{\partial \tau} & =0 \\
\operatorname{Re} \frac{\partial}{\partial \tau} \int_{0}^{H} \bar{u} d \eta & =-H \frac{\partial \bar{P}}{\partial \theta}+\Delta \bar{\tau}_{\theta \eta}, \\
\operatorname{Re} \frac{\partial}{\partial \tau} \int_{0}^{H} \bar{w} d \eta & =-H \frac{\partial \bar{P}}{\partial \xi}+\Delta \bar{\tau}_{\xi \eta} .
\end{aligned}
$$

The dimensionless lubricant average flows are defined by the following:

$$
\overline{q_{i}}=\int_{0}^{H} \bar{u}_{i} d \eta
$$

Substituting (16) into (15) gives the following:

$$
\begin{aligned}
\frac{\partial \overline{q_{\theta}}}{\partial \theta}+\frac{\partial \overline{q_{\xi}}}{\partial \xi}+\frac{\partial H}{\partial \tau}= & 0 \\
\operatorname{Re} \frac{\partial \overline{q_{\theta}}}{\partial \tau} & =-H \frac{\partial \bar{P}}{\partial \theta}+\Delta \bar{\tau}_{\theta \eta}, \\
\operatorname{Re} \frac{\partial \overline{q_{\xi}}}{\partial \tau} & =-H \frac{\partial \bar{P}}{\partial \xi}+\Delta \bar{\tau}_{\xi \eta} .
\end{aligned}
$$

Assuming that the shapes of the velocity profiles are not strongly influenced by the inertia forces [13], the wall shear stress differences can be expressed by the following:

$$
\begin{gathered}
\Delta \bar{\tau}_{\theta \eta}=-\frac{12}{H^{2}} \overline{q_{\theta}}, \\
\Delta \bar{\tau}_{\xi \eta}=-\frac{12}{H^{2}} \overline{q_{\xi}} ;
\end{gathered}
$$

hence,

$$
\begin{aligned}
\frac{\partial \overline{q_{\theta}}}{\partial \theta}+\frac{\partial \overline{q_{\xi}}}{\partial \xi}+\frac{\partial H}{\partial \tau} & =0, \\
\operatorname{Re} \frac{\partial \overline{q_{\theta}}}{\partial \tau} & =-H \frac{\partial \bar{P}}{\partial \theta}-\frac{12}{H^{2}} \overline{q_{\theta}}, \\
\operatorname{Re} \frac{\partial \overline{q_{\xi}}}{\partial \tau} & =-H \frac{\partial \bar{P}}{\partial \xi}-\frac{12}{H^{2}} \overline{q_{\xi}} .
\end{aligned}
$$


Furthermore, assuming that the temporal inertia terms in the above equations are approximated by using inertialess and dimensionless average flows [24], the equations become

$$
\begin{aligned}
& \operatorname{Re} \frac{\partial \overline{q_{\theta_{0}}}}{\partial \tau}=-H \frac{\partial \bar{P}}{\partial \theta}-\frac{12}{H^{2}} \overline{q_{\theta}}, \\
& \operatorname{Re} \frac{\partial \overline{\bar{q}_{0}}}{\partial \tau}=-H \frac{\partial \bar{P}}{\partial \xi}-\frac{12}{H^{2}} \overline{q_{\xi}},
\end{aligned}
$$

where based on Reynolds equation

$$
\begin{aligned}
& \overline{q_{\theta_{0}}}=-\frac{H^{3}}{12} \frac{\partial \overline{P_{0}}}{\partial \theta}, \\
& \overline{q_{\xi_{0}}}=-\frac{H^{3}}{12} \frac{\partial \overline{P_{0}}}{\partial \xi} .
\end{aligned}
$$

Substituting (23) into (20) and (21) gives the following:

$$
\begin{aligned}
& \operatorname{Re} \frac{\partial}{\partial \tau}\left(-\frac{H^{3}}{12} \frac{\partial \overline{P_{0}}}{\partial \theta}\right)=-H \frac{\partial \bar{P}}{\partial \theta}-\frac{12}{H^{2}} \overline{q_{\theta}} \\
& \operatorname{Re} \frac{\partial}{\partial \tau}\left(-\frac{H^{3}}{12} \frac{\partial \overline{P_{0}}}{\partial \xi}\right)=-H \frac{\partial \bar{P}}{\partial \xi}-\frac{12}{H^{2}} \overline{q_{\xi}}
\end{aligned}
$$

Equations (24) are rearranged to solve for the flows:

$$
\begin{aligned}
& \overline{q_{\theta}}=-\frac{H^{3}}{12} \frac{\partial \bar{P}}{\partial \theta}-\frac{H^{2}}{12} \operatorname{Re} \frac{\partial}{\partial \tau}\left(-\frac{H^{3}}{12} \frac{\partial \overline{P_{0}}}{\partial \theta}\right) \\
& \overline{q_{\xi}}=-\frac{H^{3}}{12} \frac{\partial \bar{P}}{\partial \xi}-\frac{H^{2}}{12} \operatorname{Re} \frac{\partial}{\partial \tau}\left(-\frac{H^{3}}{12} \frac{\partial \overline{P_{0}}}{\partial \xi}\right) .
\end{aligned}
$$

Substituting (25) into the continuity equation (19) gives

$$
\frac{\partial}{\partial \theta}\left(H^{3} \frac{\partial \bar{P}}{\partial \theta}\right)+\frac{\partial}{\partial \xi}\left(H^{3} \frac{\partial \bar{P}}{\partial \xi}\right)=12 \frac{\partial H}{\partial \tau}+G(\theta, \xi)
$$

where

$$
\begin{aligned}
G(\theta, \xi)= & \frac{\partial}{\partial \theta}\left[H^{2} \operatorname{Re} \frac{\partial}{\partial \tau}\left(\frac{H^{3}}{12} \frac{\partial \overline{P_{0}}}{\partial \theta}\right)\right] \\
& +\frac{\partial}{\partial \xi}\left[H^{2} \operatorname{Re} \frac{\partial}{\partial \tau}\left(\frac{H^{3}}{12} \frac{\partial \overline{P_{0}}}{\partial \xi}\right)\right] .
\end{aligned}
$$

The above equation is expanded and rearranged as follows:

$$
\begin{aligned}
& G(\theta, \xi) \\
& =\frac{\partial}{\partial \theta}\left(H^{2} \operatorname{Re}\right)\left[\frac{\partial}{\partial \tau}\left(\frac{H^{3}}{12} \frac{\partial \overline{P_{0}}}{\partial \theta}\right)\right] \\
& \quad+H^{2} \operatorname{Re} \frac{\partial}{\partial \tau}\left\{\frac{\partial}{\partial \theta}\left(\frac{H^{3}}{12} \frac{\partial \overline{P_{0}}}{\partial \theta}\right)+\frac{\partial}{\partial \xi}\left(\frac{H^{3}}{12} \frac{\partial \overline{P_{0}}}{\partial \xi}\right)\right\} .
\end{aligned}
$$

According to classical Reynolds equation, the inertialess (zeroth-order) pressure distribution in SFDs is described by the following:

$$
\frac{\partial}{\partial \theta}\left(\frac{H^{3}}{12} \frac{\partial \overline{P_{0}}}{\partial \theta}\right)+\frac{\partial}{\partial \xi}\left(\frac{H^{3}}{12} \frac{\partial \overline{P_{0}}}{\partial \xi}\right)=\frac{\partial H}{\partial \tau} .
$$

Substituting (29) into (28) gives the following:

$$
\begin{aligned}
G(\theta, \xi)= & \frac{\partial}{\partial \theta}\left(H^{2} \operatorname{Re}\right)\left[\frac{\partial}{\partial \tau}\left(\frac{H^{3}}{12} \frac{\partial \overline{P_{0}}}{\partial \theta}\right)\right] \\
& +H^{2} \operatorname{Re} \frac{\partial^{2} H}{\partial \tau^{2}}
\end{aligned}
$$

hence:

$$
\begin{aligned}
\frac{\partial}{\partial \theta}\left(H^{3} \frac{\partial \bar{P}}{\partial \theta}\right)+\frac{\partial}{\partial \xi}\left(H^{3} \frac{\partial \bar{P}}{\partial \xi}\right) \\
=12 \frac{\partial H}{\partial \tau}+H^{2} \operatorname{Re} \frac{\partial^{2} H}{\partial \tau^{2}} \\
+\frac{\partial}{\partial \theta}\left(H^{2} \operatorname{Re}\right)\left[\frac{\partial}{\partial \tau}\left(\frac{H^{3}}{12} \frac{\partial \overline{P_{0}}}{\partial \theta}\right)\right] .
\end{aligned}
$$

Consequently, the zeroth-order pressure distribution is calculated by using Reynolds equation and the solution is substituted into (31) to determine the total pressure distribution in SFDs. Finally, the fluid film reaction force components are determined by integrating the total pressure distribution over the journal surface:

$$
\left[\begin{array}{c}
\overline{F_{r}} \\
\overline{F_{t}}
\end{array}\right]=\int_{-L / D}^{L / D} \int_{\theta_{1}}^{\theta_{2}} \bar{P}(\theta, \xi)\left[\begin{array}{c}
\cos (\theta) \\
\sin (\theta)
\end{array}\right] d \theta d \xi
$$

where $\theta_{1}$ to $\theta_{2}$ is the positive range of the pressure distribution. In this work, the simplistic $\pi$-film cavitation model is applied where the film cavitation region is developed in half the damper circumference. The following section represents the numerical solution for the pressure distribution and fluid film reaction forces for SFDs.

\section{Numerical Solution}

This section describes the numerical technique that is applied to (31) to determine the pressure distribution and the fluid film forces in it. In order to determine the numerical solution, firstly, a solution domain is defined for the problem. Subsequently, the partial differential equations are discretized over the solution domain. Finally, an iterative numerical algorithm is developed to calculate the pointwise pressure distribution in the lubricant domain.

3.1. Zeroth-Order Pressure Solution. In order to determine the total fluid film pressure distribution, the zeroth-order pressure distribution must be first calculated. The zerothorder pressure distribution is characterized by (29). This 
equation is firstly expanded to facilitate the discretization of the equation terms:

$$
\left[3 H^{2} \frac{\partial H}{\partial \theta} \frac{\partial \overline{P_{0}}}{\partial \theta}+H^{3} \frac{\partial^{2} \overline{P_{0}}}{\partial \theta^{2}}\right]+H^{3} \frac{\partial^{2} \overline{P_{0}}}{\partial \xi^{2}}=12 \frac{\partial H}{\partial \tau}
$$

The partial derivatives of the dimensionless fluid film thickness $(H)$ are given as follows:

$$
\begin{aligned}
& \frac{\partial H}{\partial \theta}=-\varepsilon \sin (\theta), \\
& \frac{\partial H}{\partial \tau}=\dot{\varepsilon} \cos (\theta)+\varepsilon \sin (\theta) .
\end{aligned}
$$

Assuming that the SFD executes CCOs, the radial velocity and acceleration of the journal center become zero and

$$
\begin{aligned}
\frac{\partial H}{\partial \tau} & =\varepsilon \sin (\theta), \\
\frac{\partial^{2} H}{\partial \tau^{2}} & =-\varepsilon \cos (\theta) .
\end{aligned}
$$

Substituting (35) into (33) gives

$$
\begin{aligned}
& {\left[-3 \varepsilon H^{2} \sin (\theta) \frac{\partial \overline{P_{0}}}{\partial \theta}+H^{3} \frac{\partial^{2} \overline{P_{0}}}{\partial \theta^{2}}\right]+H^{3} \frac{\partial^{2} \overline{P_{0}}}{\partial \xi^{2}}} \\
& =12 \varepsilon \sin (\theta) .
\end{aligned}
$$

Subsequently, (36) is discretized based on a finite difference approximation (FDA) technique that uses a backward difference approximation for the first derivative terms and a central difference approximation for the second derivative terms, where

$$
\begin{aligned}
\frac{\partial \bar{P}}{\partial \theta} & =\frac{\bar{P}(\theta, \xi)-\bar{P}(\theta-\Delta \theta, \xi)}{\Delta \theta}=\frac{\bar{P}_{i, j}-\bar{P}_{i-1, j}}{\Delta \theta} \\
\frac{\partial \bar{P}}{\partial \xi} & =\frac{\bar{P}(\theta, \xi)-\bar{P}(\theta, \xi-\Delta \xi)}{\Delta \xi}=\frac{\bar{P}_{i, j}-\bar{P}_{i, j-1}}{\Delta \xi} \\
\frac{\partial^{2} \bar{P}}{\partial \theta^{2}} & =\frac{\bar{P}(\theta+\Delta \theta, \xi)-2 \bar{P}(\theta, \xi)+\bar{P}(\theta-\Delta \theta, \xi)}{\Delta \theta^{2}} \\
& =\frac{\bar{P}_{i+1, j}-2 \bar{P}_{i, j}+\bar{P}_{i-1, j}}{\Delta \theta^{2}}, \\
\frac{\partial^{2} \bar{P}}{\partial \xi^{2}} & =\frac{\bar{P}_{(\theta, \xi+\Delta \xi)-2 \bar{P}(\theta, \xi)+\bar{P}(\theta, \xi-\Delta \xi)}^{\Delta \xi^{2}}}{} \\
& =\frac{\bar{P}_{i, j+1}-2 \bar{P}_{i, j}+\bar{P}_{i, j-1}}{\Delta \xi^{2}} .
\end{aligned}
$$

Substituting (37) into (36) gives the discretized Reynolds equation:

$$
\begin{aligned}
& {\left[-3 \varepsilon H^{2} \sin (\theta) \frac{{\overline{P_{0 i, j}}}-\bar{P}_{0 i-1, j}}{\Delta \theta}\right.} \\
& \left.+H^{3} \frac{{\overline{P_{0 i+1, j}}}-2{\overline{P_{0 i, j}}}+{\overline{P_{0 i-1, j}}}}{\Delta \theta^{2}}\right]+H^{3} \\
& \cdot \frac{{\overline{P_{0 i, j+1}}}-2{\overline{P_{0 i, j}}}+\bar{P}_{0 i, j-1}}{\Delta \xi^{2}}=12 \varepsilon \sin (\theta) \text {. }
\end{aligned}
$$

In order to determine the pointwise pressure distribution, (38) is rearranged as follows:

$$
P_{0 i, j}=\frac{\left[12 \varepsilon \sin \theta_{i}\right]-\left[3 H_{i}^{2}\left(\varepsilon \sin \theta_{i}\right) / \Delta \theta+H_{i}^{3} / \Delta \theta^{2}\right]{\overline{P_{0 i-1, j}}}-\left[H_{i}^{3} / \Delta \theta^{2}\right]{\overline{P_{0 i+1, j}}}_{i}-\left[H_{i}^{3} / \Delta \xi^{2}\right]\left({\overline{P_{0 i, j+1}}}+{\overline{P_{0 i, j-1}}}^{2}\right)}{\left[-3 H_{i}^{2}\left(\varepsilon \sin \theta_{i}\right) / \Delta \theta-2 H_{i}^{3} / \Delta \theta^{2}-2 H_{i}^{3} / \Delta \xi^{2}\right]} .
$$

Reynolds equation is classified as an elliptical PDE. Assuming that the lubricant is incompressible and isoviscous and the journal center executes CCO whirls, the following numerical procedure is used to determine the fluid film pressure distribution for a specified SFD eccentricity ratio.

(1) The boundary points are initialized to their prescribed values and the interior points are adjusted to zero.

(2) Equation (39) is iteratively solved for the interior points.

(3) The iteration is only interrupted when the solution error reaches a convergence criterion.

(4) Finally, an overrelaxation technique is applied to accelerate the convergence of the solution:

$$
P_{0_{i, j}}^{(k)}=P_{0_{i, j}}^{(k-1)}+\lambda\left(P_{0_{i, j}}^{(k)}-P_{0_{i, j}}^{(k-1)}\right) .
$$

3.2. Total Pressure Solution. The numerical procedure that is employed to determine the total pressure distribution is very similar to the one for the zeroth-order pressure. The total pressure distribution in the fluid film is characterized by (31). This equation is first expanded to facilitate the discretization of the differential terms:

$$
\begin{aligned}
& H^{3} \frac{\partial^{2} \bar{P}}{\partial \theta^{2}}+3 H^{2} \frac{\partial H}{\partial \theta} \frac{\partial \bar{P}}{\partial \theta}+H^{3} \frac{\partial^{2} \bar{P}}{\partial \xi^{2}}=12 \frac{\partial H}{\partial \tau}+G(\theta, \xi), \\
& G(\theta, \xi) \\
& =H^{2} \operatorname{Re} \frac{\partial^{2} H}{\partial \tau^{2}} \\
& \quad+\frac{\operatorname{Re} H}{6} \frac{\partial H}{\partial \theta}\left[H^{3} \frac{\partial^{2} \overline{P_{0}}}{\partial \tau \partial \theta}+3 H^{2} \frac{\partial H}{\partial \tau} \frac{\partial \overline{P_{0}}}{\partial \theta}\right],
\end{aligned}
$$


where

$$
\frac{\partial^{2} \overline{P_{0}}}{\partial \tau \partial \theta}=\frac{\partial}{\partial \tau}\left(\frac{\partial \overline{P_{0}}}{\partial \theta}\right)=\frac{\partial}{\partial \theta}\left(\frac{\partial \overline{P_{0}}}{\partial \theta}\right) \frac{\partial \theta}{\partial \tau}=-\frac{\partial^{2} \overline{P_{0}}}{\partial \theta^{2}} .
$$

Equations (41) are discretized by using (37) and the partial derivatives of the fluid film thickness for CCO in (35) are substituted into the following equations:

$$
\begin{aligned}
& H^{3} \frac{\bar{P}_{i+1, j}-2 \bar{P}_{i, j}+\bar{P}_{i-1, j}}{\Delta \theta^{2}}-3 H^{2} \varepsilon \sin (\theta) \frac{\bar{P}_{i, j}-\bar{P}_{i-1, j}}{\Delta \theta}+H^{3} \frac{\bar{P}_{i, j+1}-2 \bar{P}_{i, j}+\bar{P}_{i, j-1}}{\Delta \xi^{2}}=12 \varepsilon \sin (\theta)+G_{i, j}, \\
& G_{i, j}=-H^{2} \operatorname{Re} \varepsilon \cos (\theta)-\frac{\operatorname{Re} H}{6} \varepsilon \sin (\theta)\left[3 H^{2} \varepsilon \sin (\theta) \frac{\bar{P}_{0 i, j}-\overline{P_{0 i-1, j}}}{\Delta \theta}-H^{3} \frac{\bar{P}_{0 i, j+1}-2 \bar{P}_{0 i, j}+\bar{P}_{0 i, j-1}}{\Delta \xi^{2}}\right], \\
& P_{i, j}=\frac{\left[12 \varepsilon \sin \theta_{i}+G_{i, j}\right]-\left[3 H_{i}^{2}\left(\varepsilon \sin \theta_{i}\right) / \Delta \theta+H_{i}^{3} / \Delta \theta^{2}\right] \bar{P}_{i-1, j}-\left[H_{i}^{3} / \Delta \theta^{2}\right] \bar{P}_{i+1, j}-\left[H_{i}^{3} / \Delta \xi^{2}\right]\left(\bar{P}_{i, j+1}+\bar{P}_{i, j-1}\right)}{\left[-3 H_{i}^{2}\left(\varepsilon \sin \theta_{i}\right) / \Delta \theta-2 H_{i}^{3} / \Delta \theta^{2}-2 H_{i}^{3} / \Delta \xi^{2}\right]} .
\end{aligned}
$$

Subsequently, (45) is iteratively solved by using the numerical procedure that is described in the previous section. Finally, the fluid film reaction forces are determined by numerically integrating the pressure field over the journal surface:

$$
\left[\begin{array}{l}
\overline{F_{r}} \\
\overline{F_{t}}
\end{array}\right]=\sum_{i=1}^{N} \sum_{j=1}^{M} \bar{P}_{i, j}\left[\begin{array}{l}
\cos \left(\theta_{i}\right) \\
\sin \left(\theta_{i}\right)
\end{array}\right] \Delta \theta \Delta \xi
$$

\section{Results and Discussion}

This section represents the simulation results for the pressure distribution and the fluid film reaction forces for the proposed hydrodynamic SFD model, under different operating conditions, including journal eccentricity ratios, journal slenderness ratios, and Reynolds numbers (i.e., inertia effects). The numerical algorithm that was developed in the previous section is incorporated into Matlab to evaluate the effect of SFD operating parameters on the lubricant pressure distribution and the fluid film reaction forces. In order to validate the proposed model, the results are compared against the existing SFD models. San Andres and Vance [9] have proposed a linearized force coefficient model for SFDs executing small amplitude circular-centered orbits. They proposed a closedform analytical model for the SFD pressure distribution as follows:

$$
\bar{P}=\varepsilon \operatorname{Re} Z(\xi)\left[k_{1} \cos \theta-k_{2} \sin \theta\right]
$$

where

$$
Z(\xi)=1-\frac{\cosh (\xi)}{\cosh (L / D)}
$$

and $k_{1}$ and $k_{2}$ are the real and imaginary parts of $k$, which is defined as follows:

$$
k=\frac{\zeta \sinh (\zeta)}{[\zeta \sinh (\zeta)+2-2 \cosh (\zeta)]},
$$

where

$$
\zeta=(1-i) \sqrt{\frac{\mathrm{Re}}{2}} .
$$

Figure 3 represents the effect of fluid inertia on the dimensionless fluid pressure distribution at the bearing axial midplane $(\xi=0)$ at small eccentricity ratios for the numerical solution based on (45), the analytical pressure expression based on (47), and Reynolds equation (i.e., no inertia effect) in (39). In general, the fluid inertia effect causes a significant increase in the pressure amplitude, a change in the shape of the pressure profile, and a phase shift of the pressure peak in the direction of the journal precession. Furthermore, at small Reynolds numbers, the influence of the viscous forces makes the pressure profile closer to a sinusoid; however, at moderate and large Reynolds numbers the pressure is in phase with the gap acceleration and transforms into a cosine wave shape.

Figure 4 illustrates the effect of journal eccentricity ratio on the dimensionless midplane pressure distribution at moderate inertia effects (i.e., moderate Reynolds number). According to the results, the pressure amplitude considerably increases with the journal eccentricity ratio. Furthermore, the shape and the phase of the pressure profile are not considerably influenced by the eccentricity ratio.

Figure 5 demonstrates the effect of journal slenderness ratio on the dimensionless midplane pressure profile at small eccentricity ratios and moderate Reynolds numbers. Increasing the slenderness ratio significantly increases the pressure amplitude; however, the shape and the phase of the pressure profile are unaffected.

Furthermore, Vance [25] has developed the force coefficients for the short-length SFD with open ends by using the simplistic $\pi$-film assumption (i.e., film cavitation region is developed in half the damper circumference). The force coefficients are represented as follows:

$$
\begin{aligned}
& C_{t t}=\frac{\pi \mu D}{4\left(1-\varepsilon^{2}\right)^{3 / 2}}\left(\frac{L}{c}\right)^{3}, \\
& C_{r t}=\frac{\mu \varepsilon D}{\left(1-\varepsilon^{2}\right)^{2}}\left(\frac{L}{c}\right)^{3},
\end{aligned}
$$




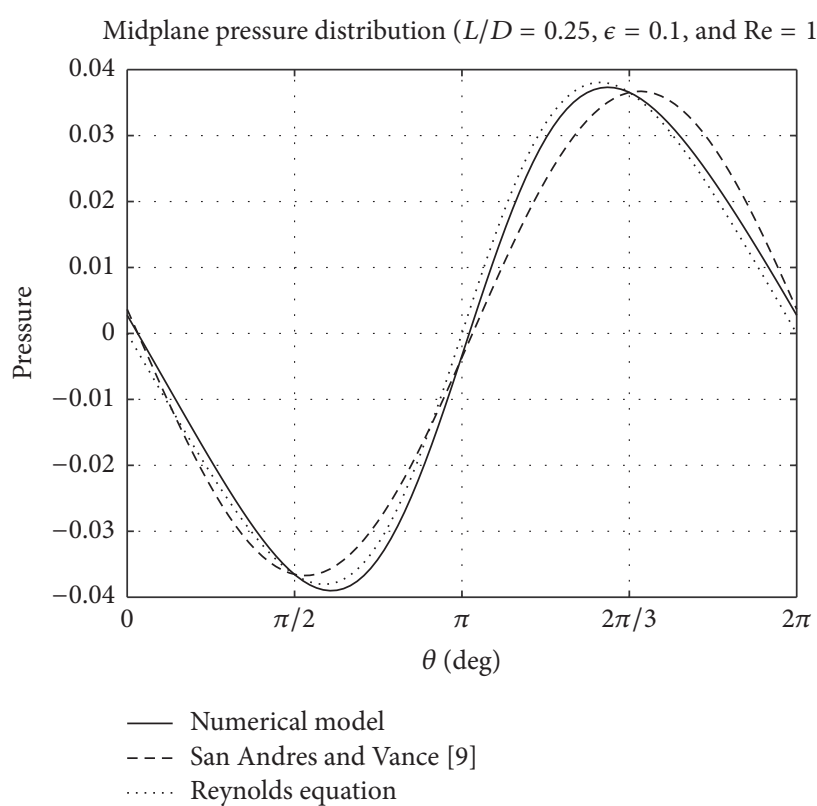

(a)

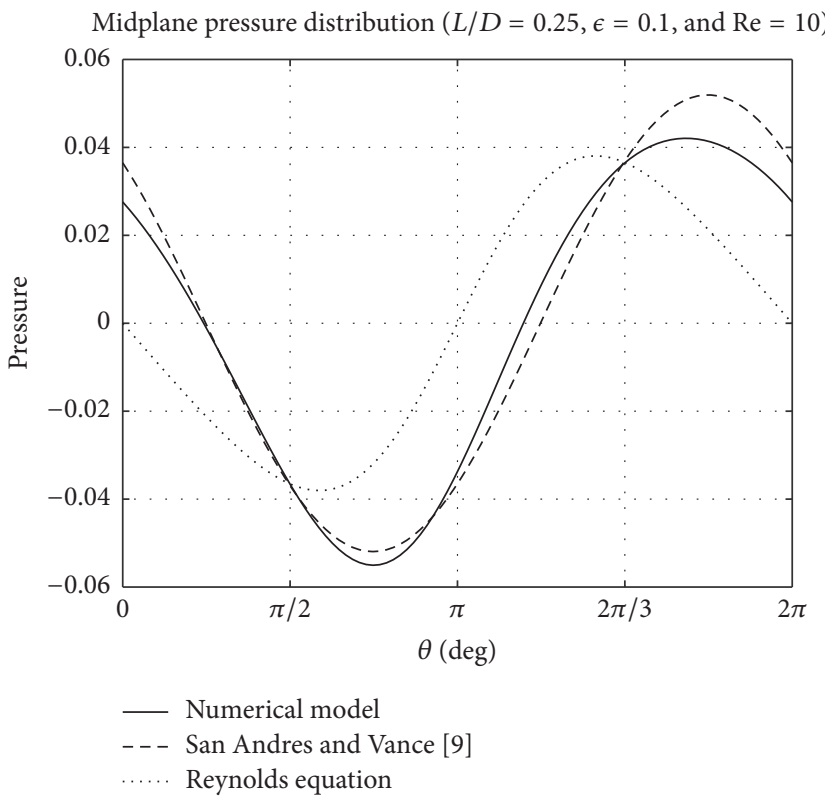

(c)

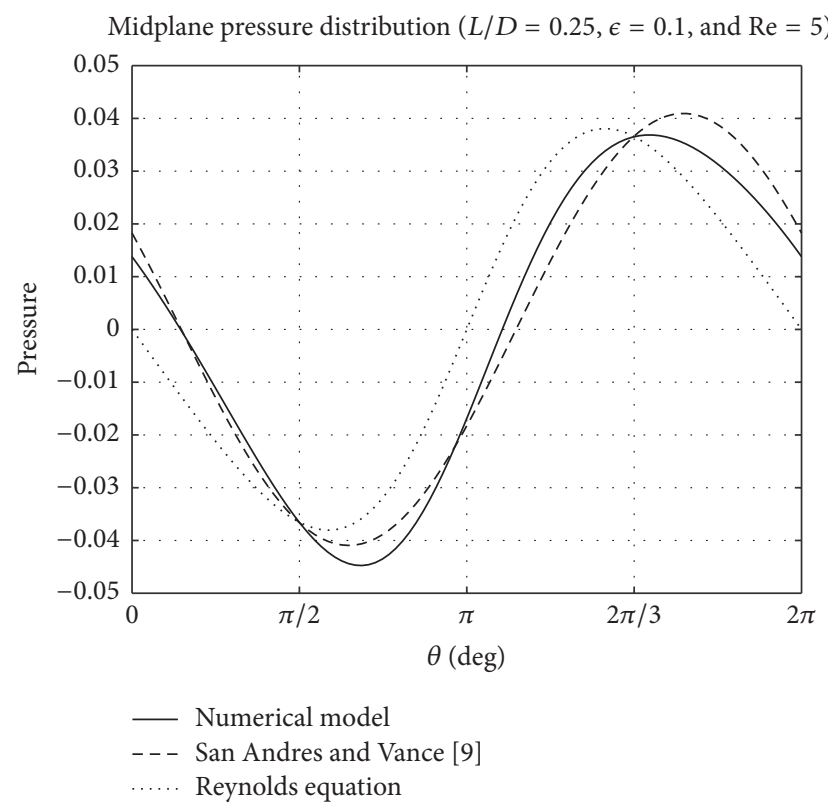

(b)

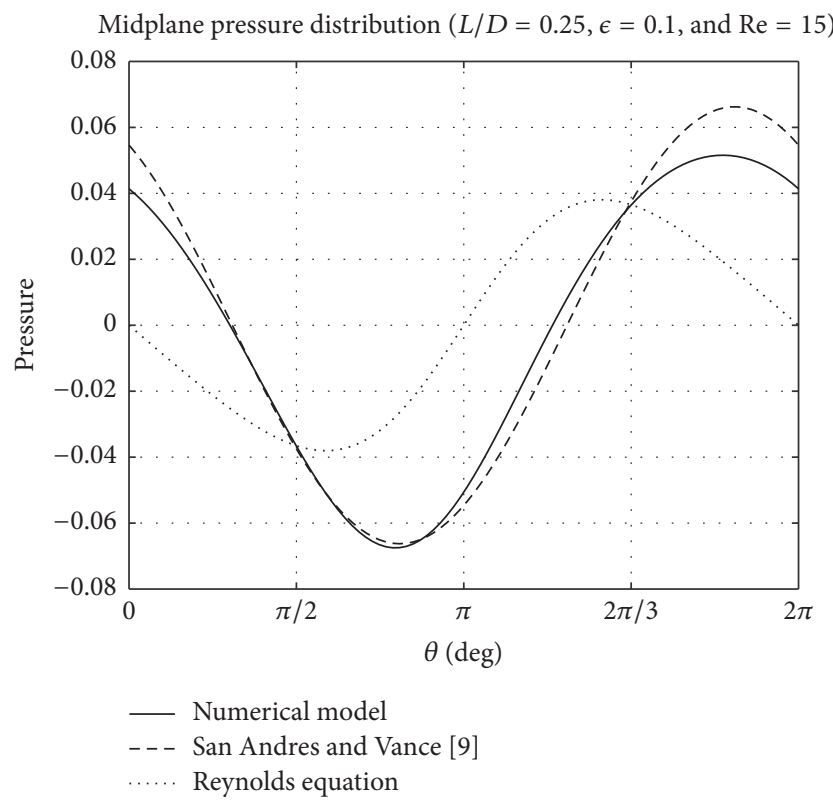

(d)

FIGURE 3: The effect of fluid inertia on the dimensionless pressure distribution.

$$
\begin{aligned}
M_{r r} & =\frac{\pi \rho D}{24}\left(\frac{L^{3}}{c}\right)\left[1-2\left(1-\varepsilon^{2}\right)^{1 / 2}\right] \\
& \left\{\frac{\left(1-\varepsilon^{2}\right)^{1 / 2}-1}{\varepsilon^{2}\left(1-\varepsilon^{2}\right)^{1 / 2}}\right\}, \\
M_{t r} & =-\frac{27 \rho D}{140 \varepsilon}\left(\frac{L^{3}}{c}\right)\left[2+\frac{1}{\varepsilon} \ln \left(\frac{1-\varepsilon}{1+\varepsilon}\right)\right] .
\end{aligned}
$$

The fluid film reaction force components are calculated based on the force coefficients in (51) to (54) as follows:

$$
\begin{aligned}
& F_{r}=-\left(C_{r t} V_{t}+M_{r r} A_{r}\right), \\
& F_{r}=-\left(C_{t t} V_{t}+M_{t r} A_{r}\right),
\end{aligned}
$$

where

$$
\begin{aligned}
& V_{t}=\varepsilon c \omega, \\
& A_{r}=-\varepsilon c \omega^{2},
\end{aligned}
$$




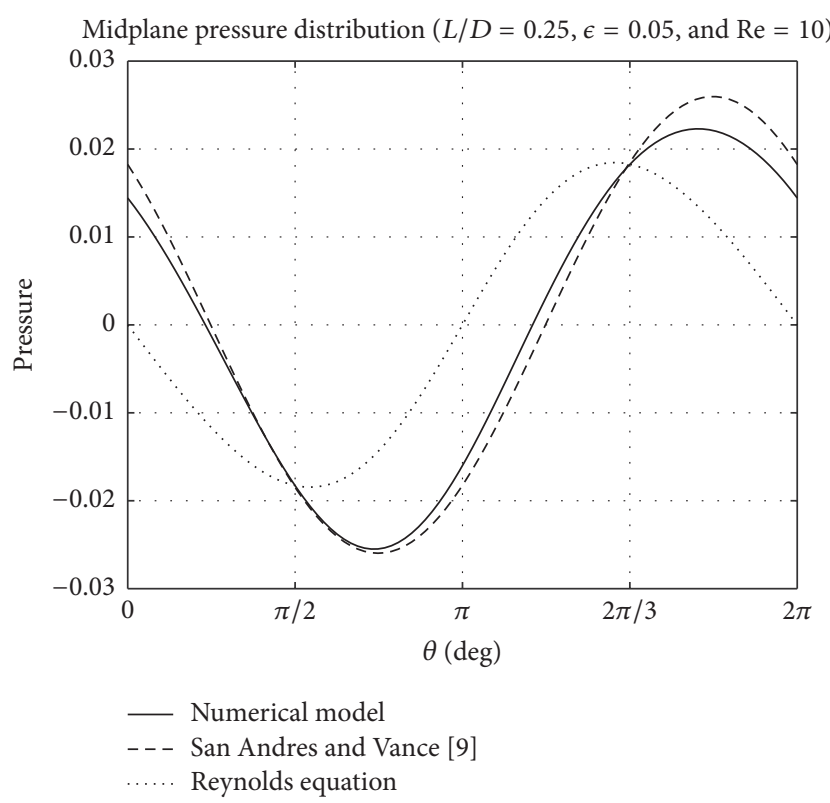

(a)

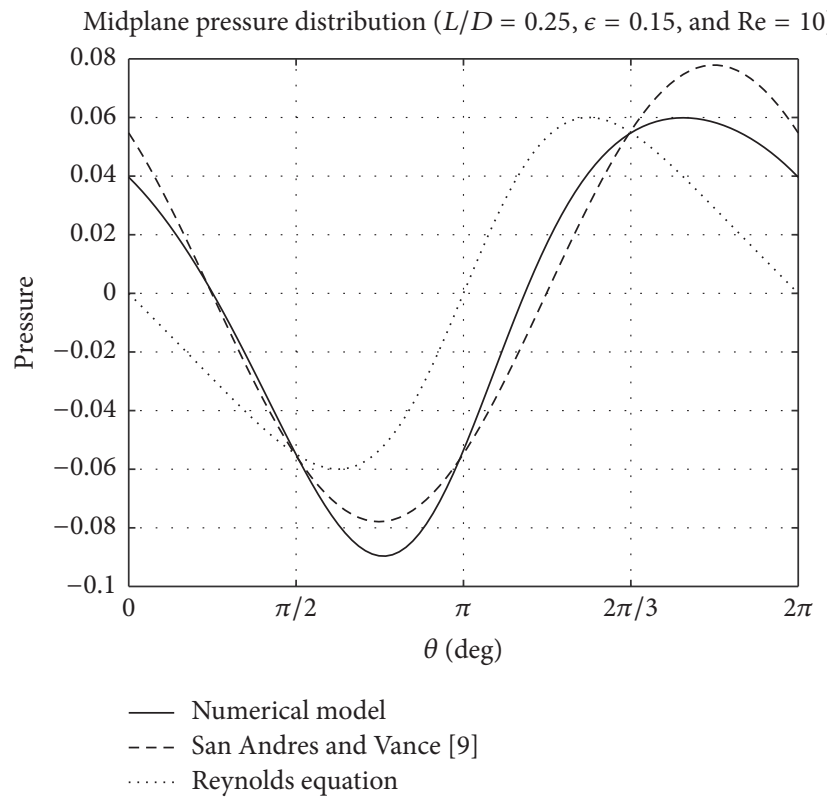

(c)

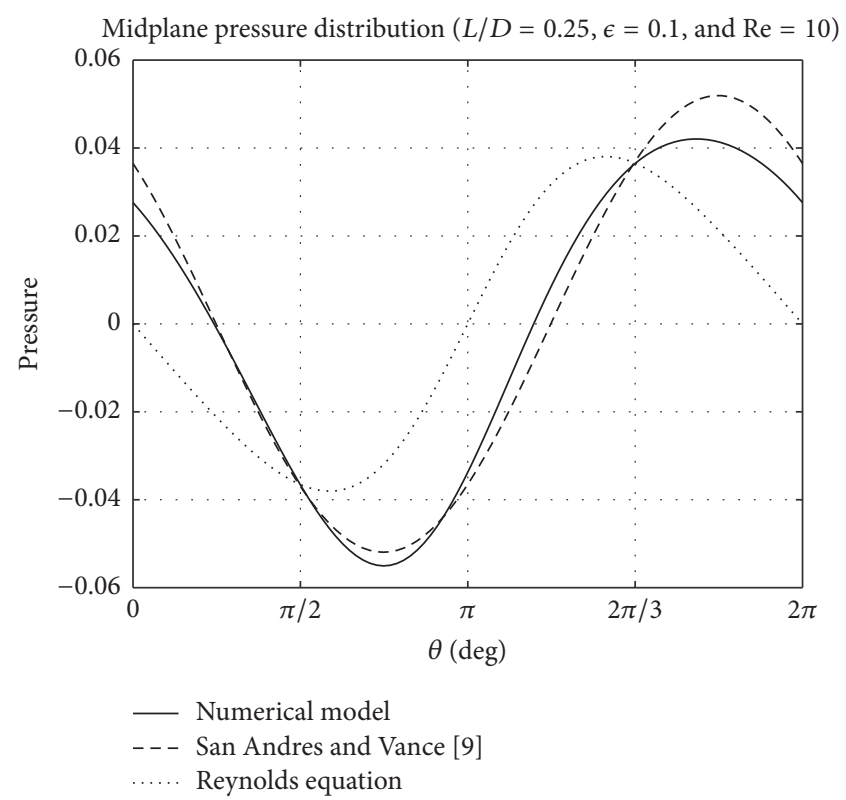

(b)

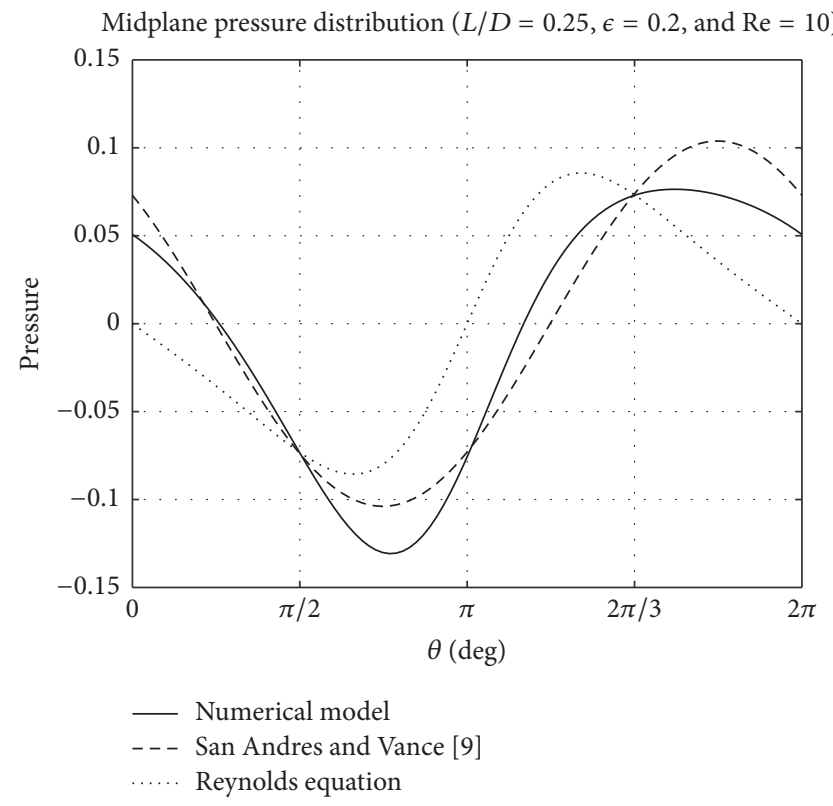

(d)

FIGURE 4: The effect of SFD journal eccentricity ratio on the dimensionless pressure distribution.

$$
\begin{aligned}
& \overline{F_{r}}=\frac{C_{f}}{F_{r}}, \\
& \overline{F_{t}}=\frac{C_{f}}{F_{t}},
\end{aligned}
$$

where

$$
C_{f}=\frac{\mu \omega R^{4}}{c^{2}}
$$

Subsequently, the fluid film reaction forces for the proposed model are compared against the results for San Andres and
Vance [9] and Vance [25]. Figure 6 represents the effect of fluid inertia on the SFD fluid film reaction forces at small eccentricity ratios. The fluid forces are calculated by numerically integrating the lubricant hydrodynamic pressure distribution over the journal surface. The magnitude and the direction of the radial fluid film forces change with the fluid inertia effects. For SFDs executing small amplitude CCOs, the effect of fluid inertia is characterized by using a temporal inertia component. At no inertia effects (i.e., $\mathrm{Re}=0$ ) the effect of temporal inertia is neglected and the fluid film is characterized by Reynolds equation. In this case, the fluid film radial forces are negative, meaning that the direction of the 


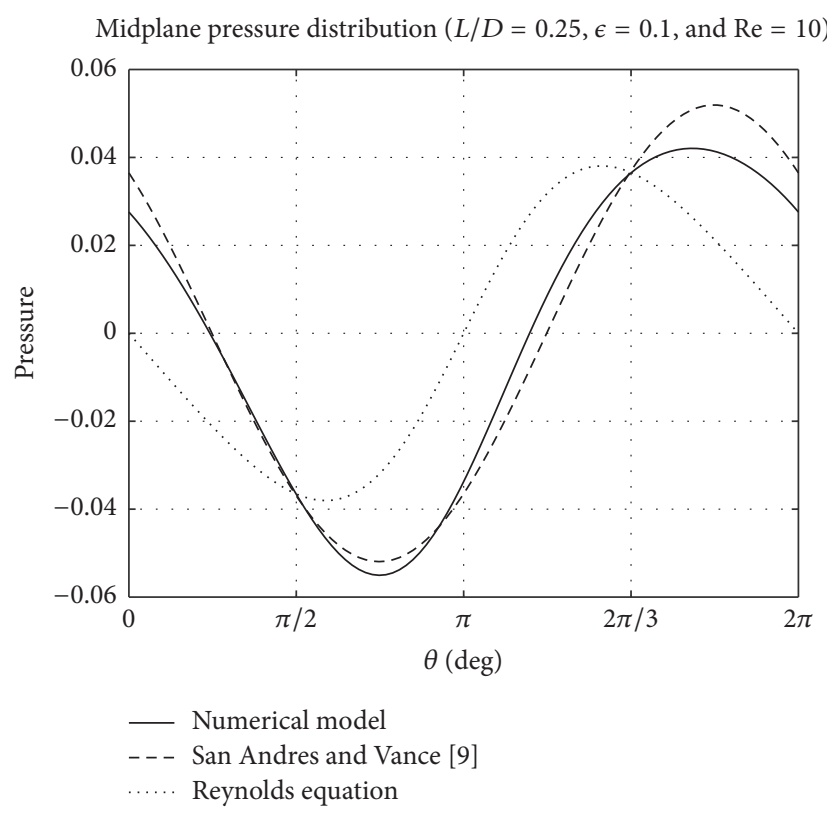

(a)

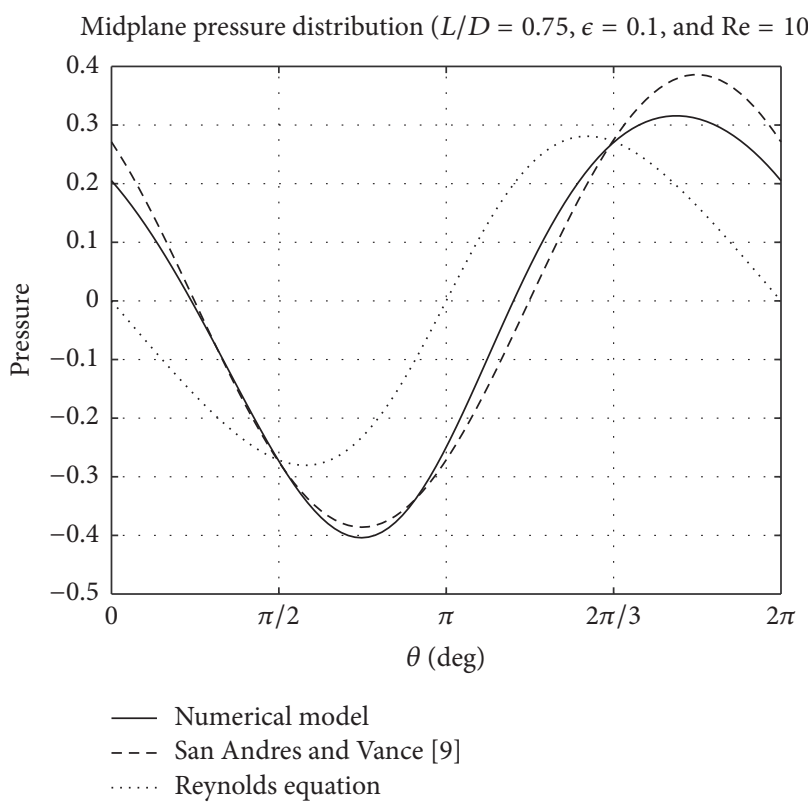

(c)

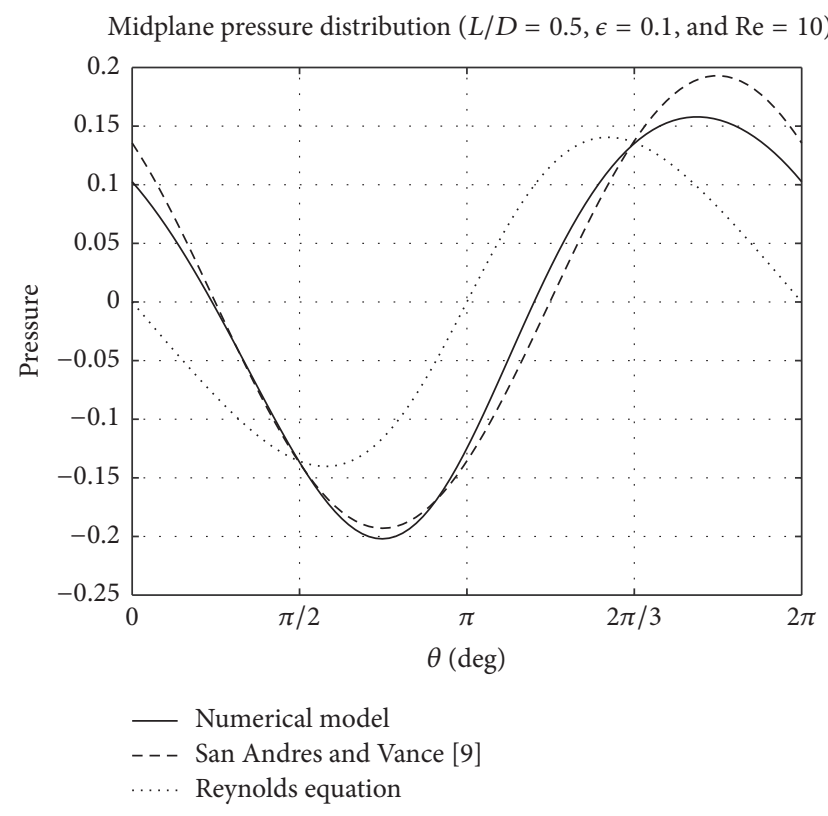

(b)

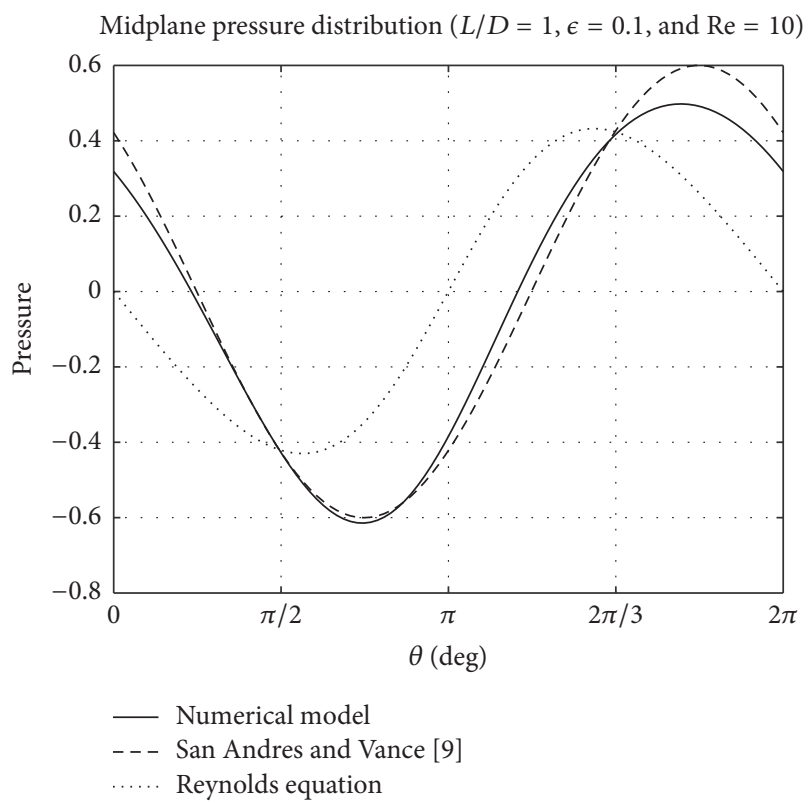

(d)

FIGURE 5: The effect of SFD journal slenderness ration on the dimensionless pressure distribution.

forces is towards the bearing center. At small Reynolds numbers (Figure 6(a)) the radial forces are positive at very small eccentricity ratios, meaning that the direction of the forces is outwards; however, as the eccentricity ratio grows slightly, the direction of the forces switches back to inwards. At moderate to large Reynolds numbers, the radial forces are positive over the range of the small eccentricity ratios and the direction of the forces stays outwards. Furthermore, at small eccentricity ratios, the magnitude of the radial forces is larger relative to the inertialess forces, meaning that the positive inertial forces dominate the negative viscous forces. Additionally, the tangential fluid film forces are not considerably influenced by fluid inertia at small Reynolds numbers. However, at larger Reynolds numbers, the magnitude of the tangential forces is slightly smaller relative to the inertialess forces. Moreover, the comparison between the numerical model and the analytical model in [9] firstly shows that the analytical model is incapable of predicting the fluid film radial forces at small Reynolds numbers, since the radial forces linearly increase with both $\operatorname{Re}$ and $\varepsilon$. Additionally, the disagreement between the results is smaller at very small eccentricity ratios and this error significantly increases for larger eccentricity 


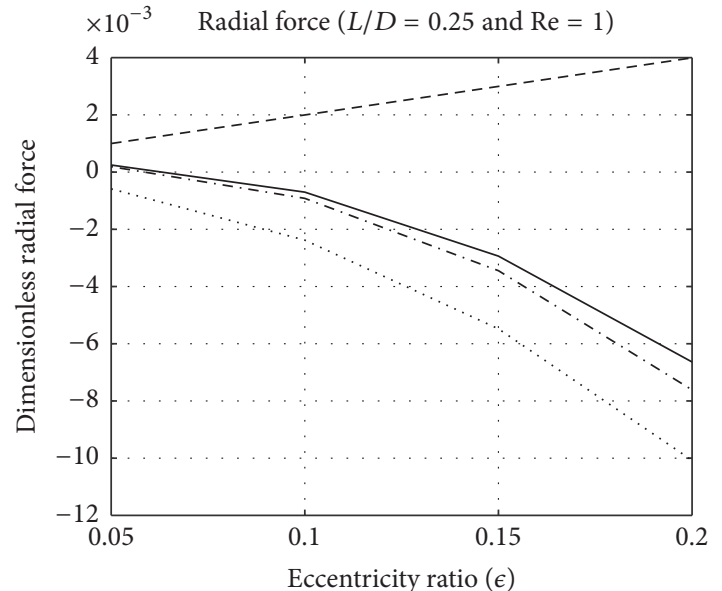

- Numerical model _..... Reynolds equation -. - San Andres and Vance [9] - ..- Vance [25]

(a)

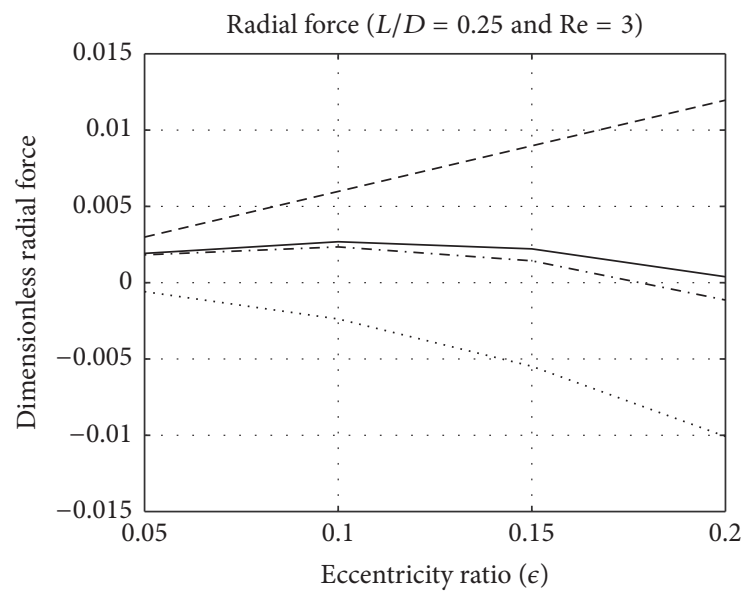

- Numerical model …... Reynolds equation - - - San Andres and Vance [9] . .. Vance [25]

(c)

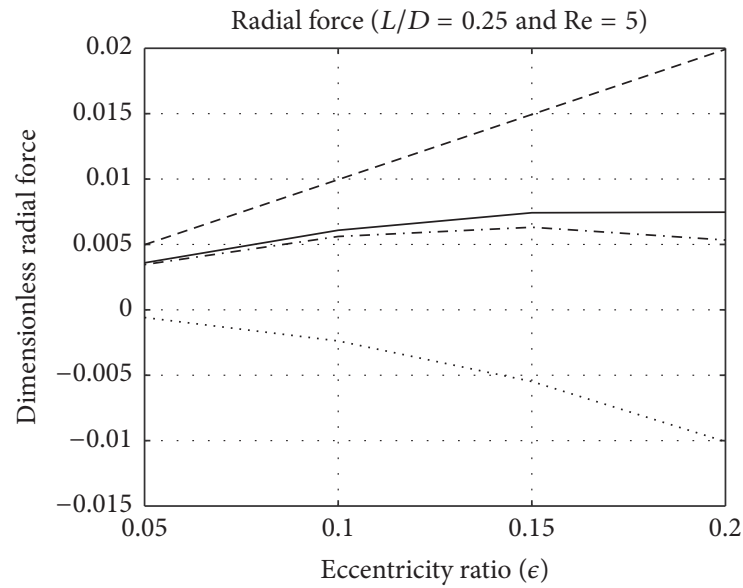

- Numerical model

...... Reynolds equation

- - - San Andres and Vance [9]

-..- Vance [25]

(e)

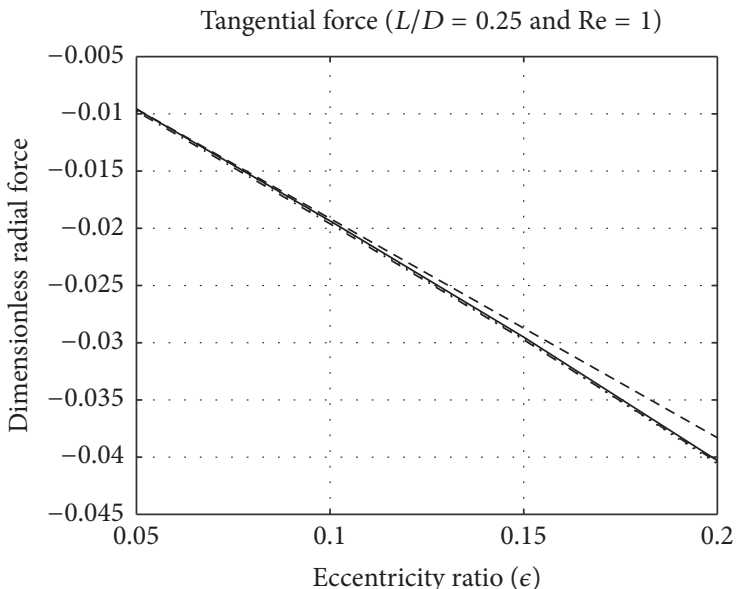

$\begin{array}{lll}\text { —- Numerical model } & \text {..... } & \text { Reynolds equation } \\ \text { - San Andres and Vance [9] } & \text {-.- } & \text { Vance [25] }\end{array}$

(b)

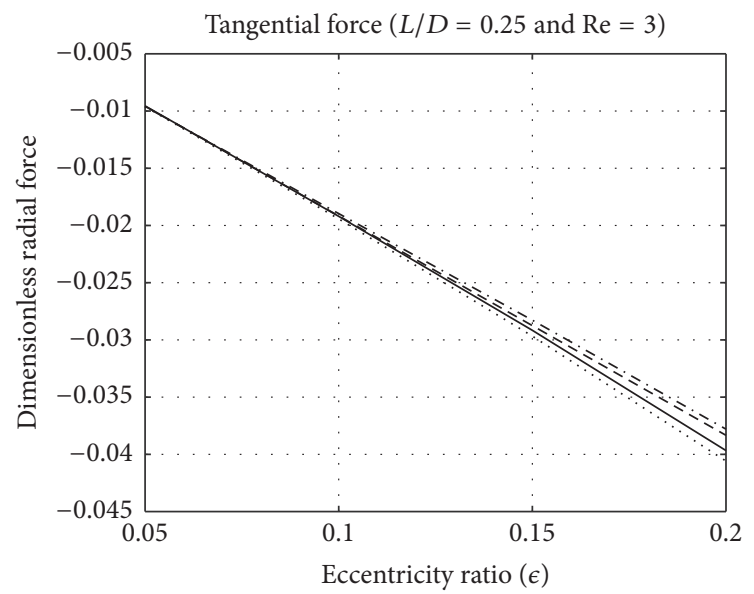

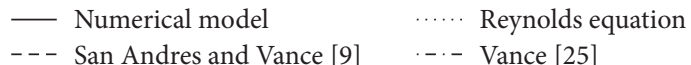

(d)

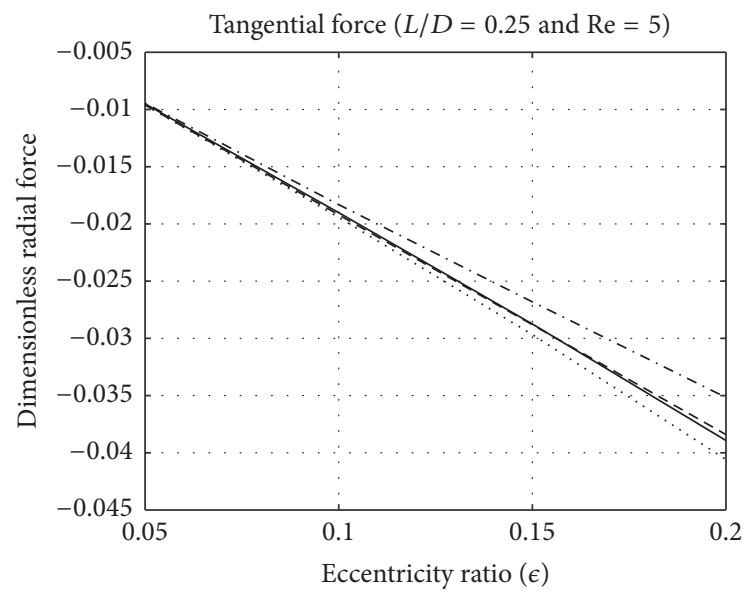

- Numerical model _..... Reynolds equation _. - San Andres and Vance [9] _... Vance [25]

(f)

Figure 6: Continued. 


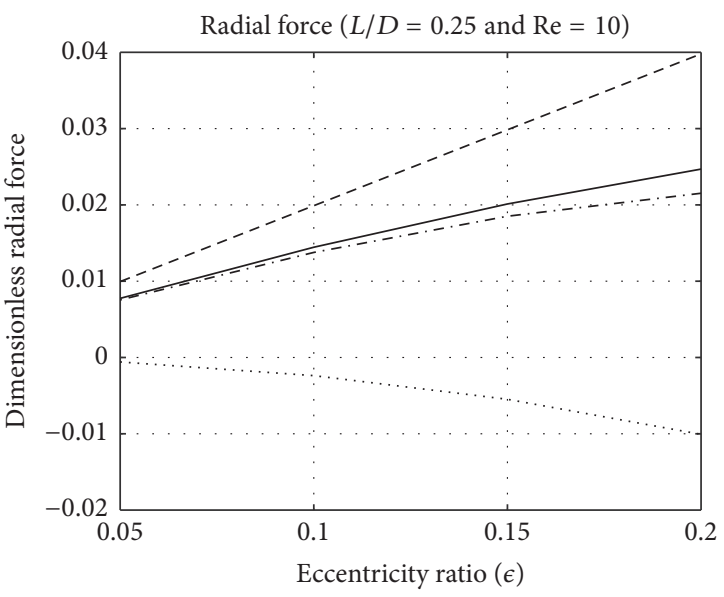

$\begin{array}{lll}\text { - Numerical model } & \text {..... } & \text { Reynolds equation } \\ \text { - - - San Andres and Vance [9] } & \text {...- } & \text { Vance [25] }\end{array}$

(g)

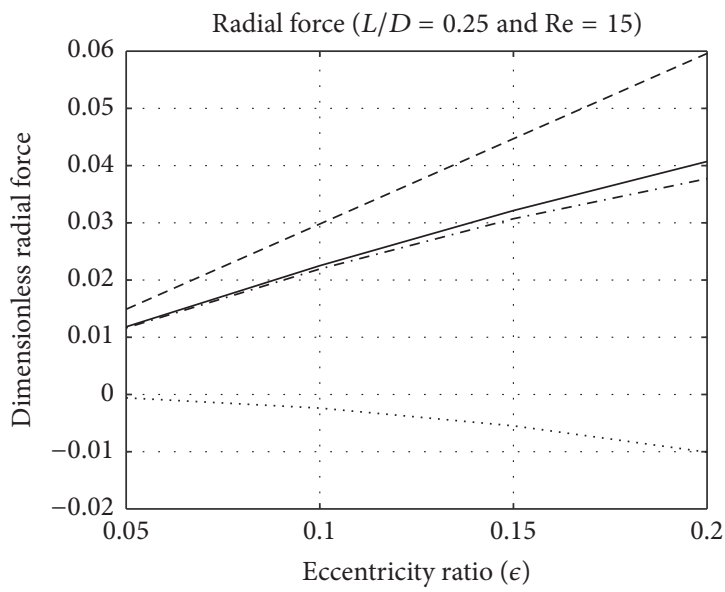

- Numerical model $\quad \cdots . .$. Reynolds equation . - - San Andres and Vance [9] . . . - Vance [25]

(i)

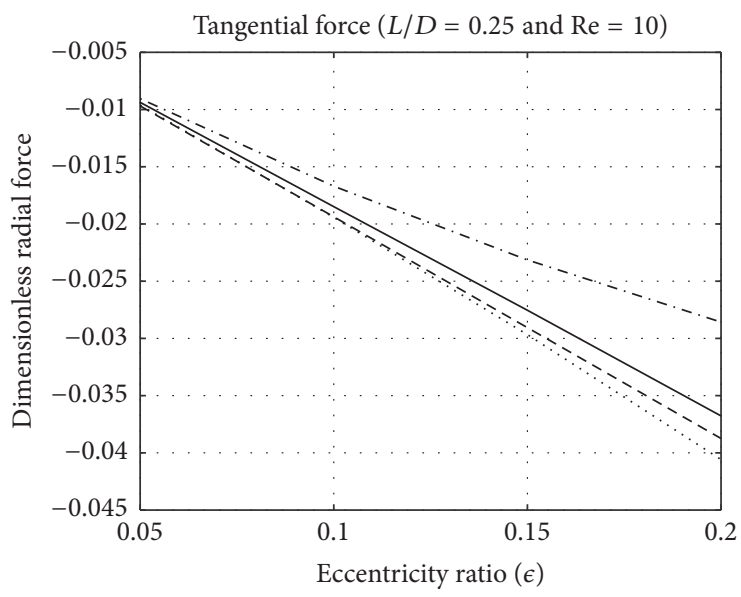

$\begin{array}{lll}\text { - Numerical model } & \ldots . . & \text { Reynolds equation } \\ \text {-- } & \text { San Andres and Vance [9] } \quad \ldots .- \text { Vance [25] }\end{array}$

(h)

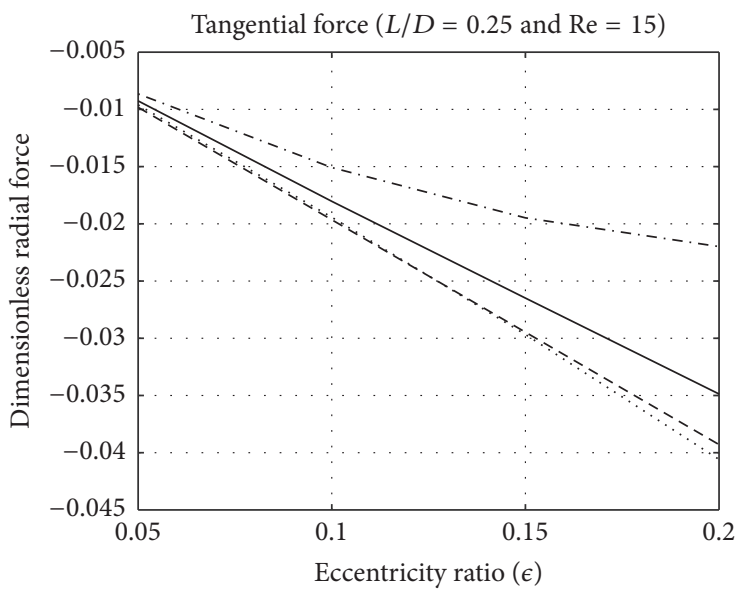

- Numerical model $\quad$...... Reynolds equation

- - - San Andres and Vance [9] ...- Vance [25]

(j)

FIGURE 6: The effect of fluid inertia on the fluid film force components for small SFD journal eccentricity ratios.

ratios, showing that [9] is valid only for very small eccentricity ratios and moderate Reynolds numbers. Furthermore, the predicted tangential forces proposed by the two models are in good agreement. Finally, the radial forces based on the numerical model demonstrate very close agreement with the analytical model in [25], especially for smaller eccentricity ratios. The tangential forces are in very close agreement for small eccentricity ratios; however, the results diverge at larger eccentricity ratio values since the analytical model is driven for short SFDs.

Figure 7 demonstrates the evolution of the numerical calculation error for the proposed numerical procedure at different eccentricity ratios. In the simulations, the solution convergence criterion is fixed at $10^{-6}$ and the iteration is interrupted once the error drops below this criterion. For all the represented calculation examples, the solution converges rapidly and the iteration is stable. Similar convergence results are expected for arbitrary SFD parameter values, including eccentricity ratios, slenderness ratios, and Reynolds numbers.

Additionally, it is typically assumed that the higher order inertia terms in the SFD pressure calculations are negligible relative to the first-order and second-order inertia terms. An important contribution of this work is including a thirdorder nonlinear term in the fluid film pressure model in (31). Including this third-order expression increases the accuracy of the pressure calculations and ultimately the fluid film forces. Figure 8 demonstrates the contribution of this term to the radial and tangential fluid film forces. The fluid film forces are calculated for two cases where (1) the third-order term is neglected and (2) the third-order expression is included, and the relative error for the reaction forces is calculated at different eccentricity ratios and inertia effects. The results of the calculations demonstrate the significant contribution of the third-order term on the accuracy of the fluid film 


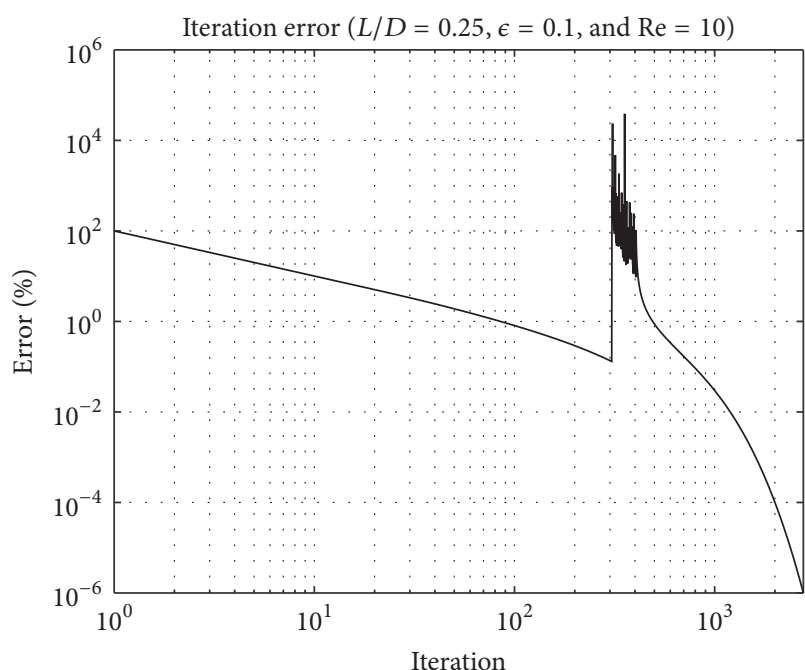

- Iteration $=2774$

(a)

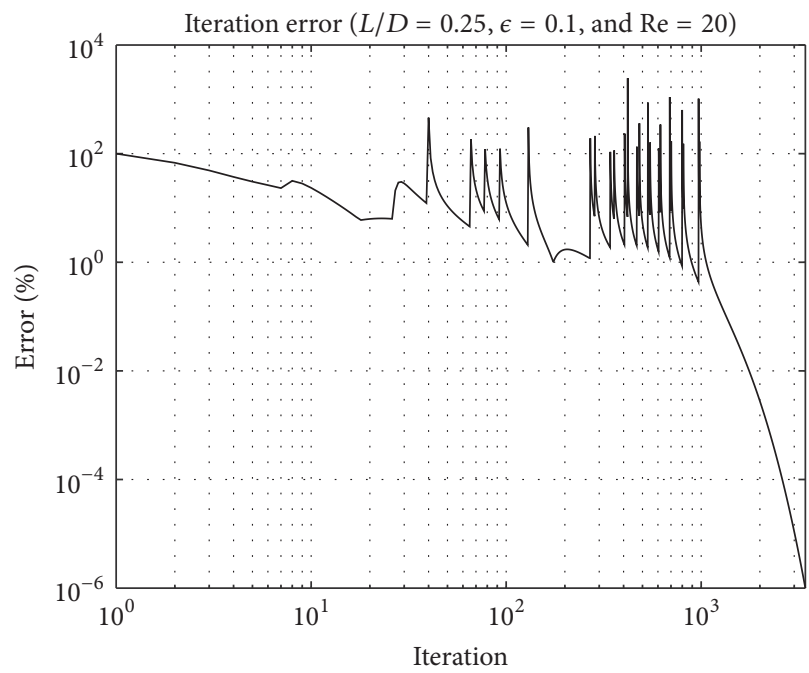

- Iteration $=3421$

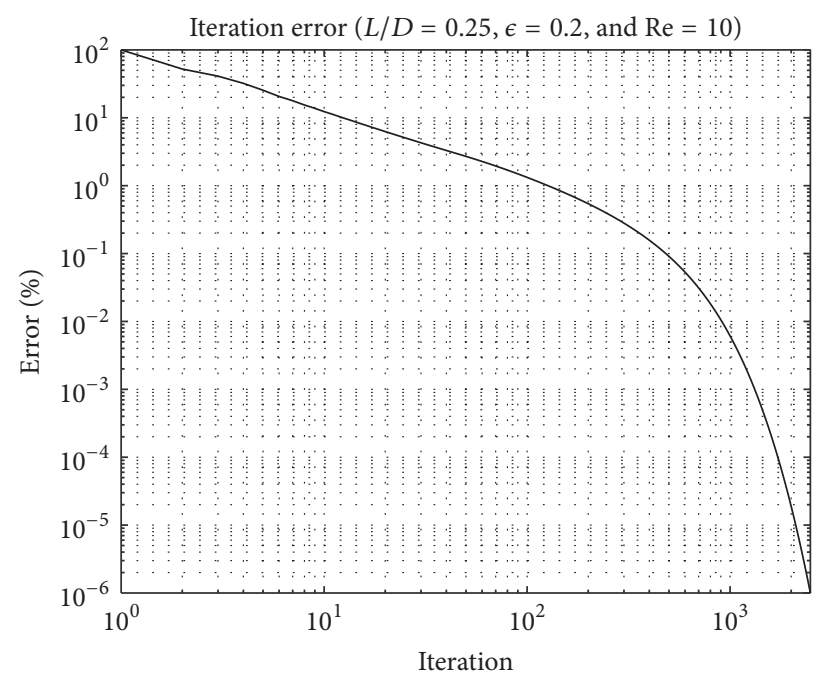

- Iteration $=2486$

(b)

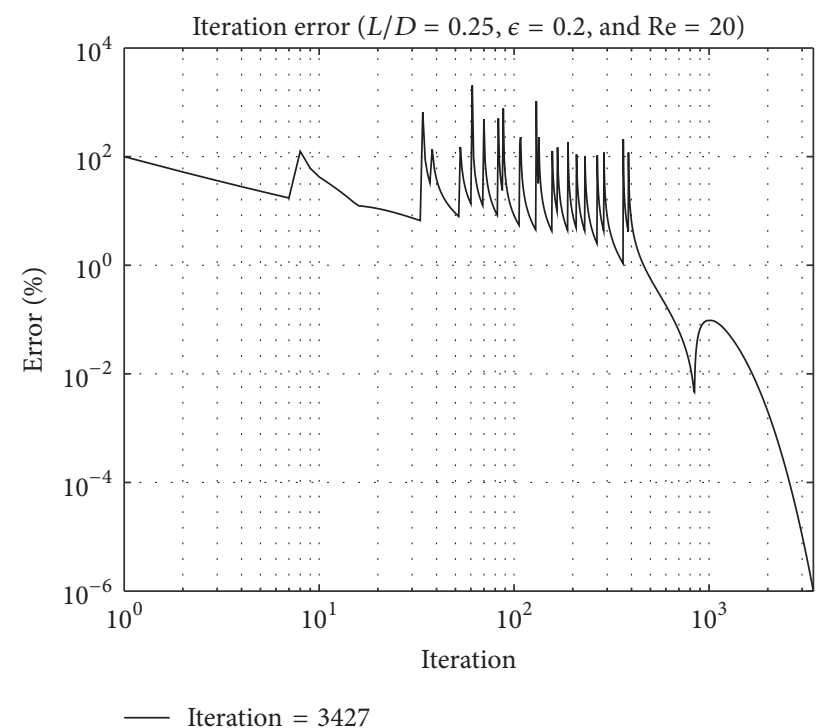

(d)

FIGURE 7: Evolution of the calculation error for several SFD operating conditions.

forces. In general, the calculation error for the radial forces declines by increasing the Reynolds number. Furthermore, the calculation error for the radial forces increases with the journal eccentricity ratio. Nevertheless, at small inertia effects (i.e., $\operatorname{Re}=1$ to $\operatorname{Re}=4$ ) the calculation error for the radial forces demonstrates irregular nonlinear behavior. The inertia effects introduce an inertial force component for the radial forces that is in the opposite direction to the viscous forces. For moderate and large Reynolds numbers the inertial forces dominate the viscous forces at small eccentricity ratios and the resultant radial forces are positive (Figures 6(e), 6(g), and 6(i)). However, at very small Reynolds numbers, the inertial forces dominate the viscous forces only over a very short range of eccentricity ratios and the radial forces quickly drop to negative (Figures 6(a) and 6(c)). This sudden variation in the radial forces results in the significant fluctuation in the calculation error. Finally, the calculation error for the tangential force increases with both Reynolds number and journal eccentricity ratio.

\section{Conclusion}

This work represented a hydrodynamic pressure distribution model for squeeze film dampers incorporated into highspeed turbomachinery. It is assumed that the journal center executes small amplitude CCO whirls, which is a very common practice in the turbomachinery industry. The proposed pressure model provides superior accuracy compared to the existing pressure models, including limiting geometry models. Moreover, a numerical procedure is proposed to solve the model for pointwise pressure distribution, which is significantly more computationally efficient relative to the 


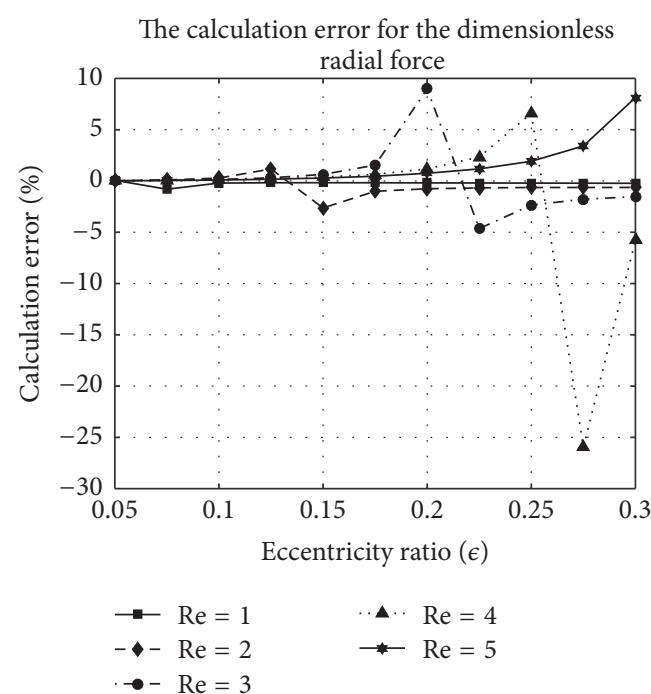

(a)

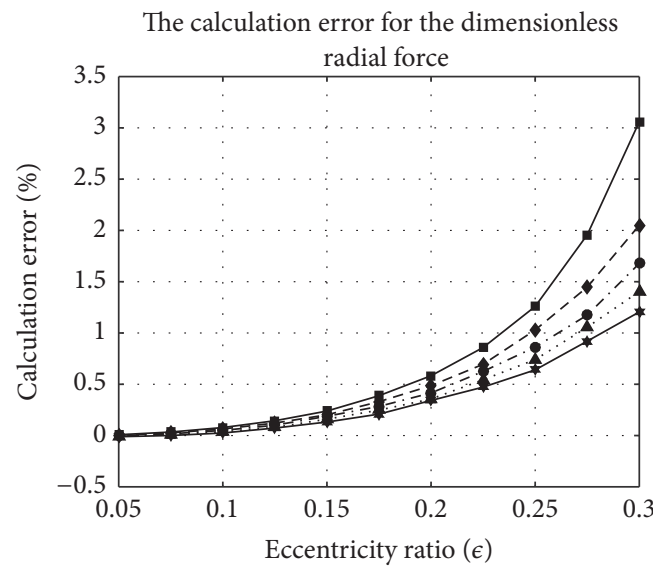

$\rightarrow \mathrm{Re}=6$

$--\operatorname{Re}=7$

- $-\mathrm{Re}=8$

(c)

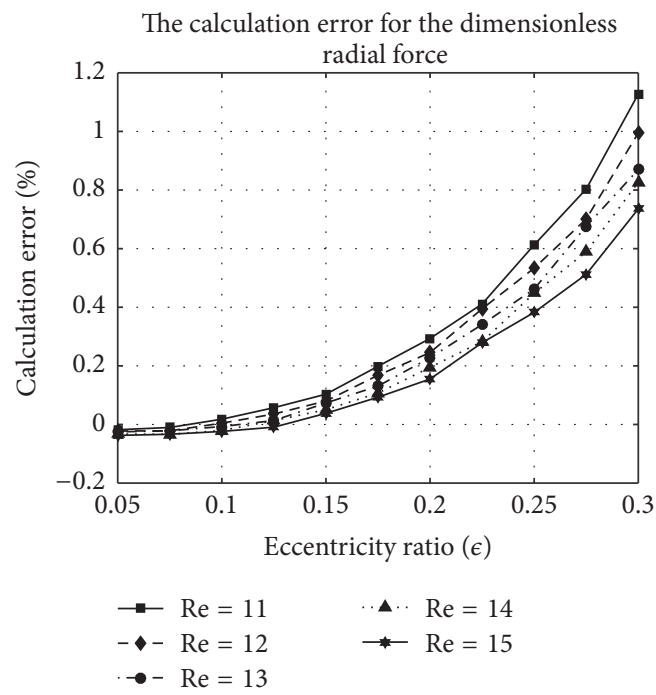

(e)

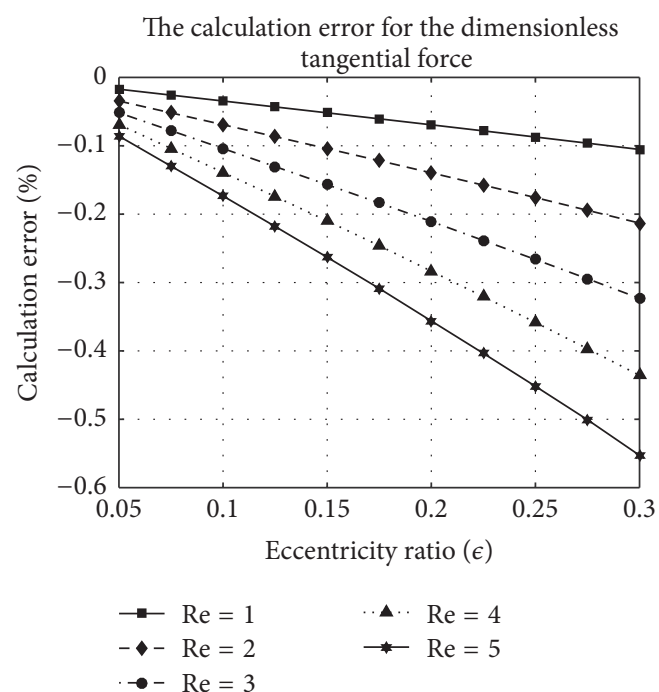

(b)
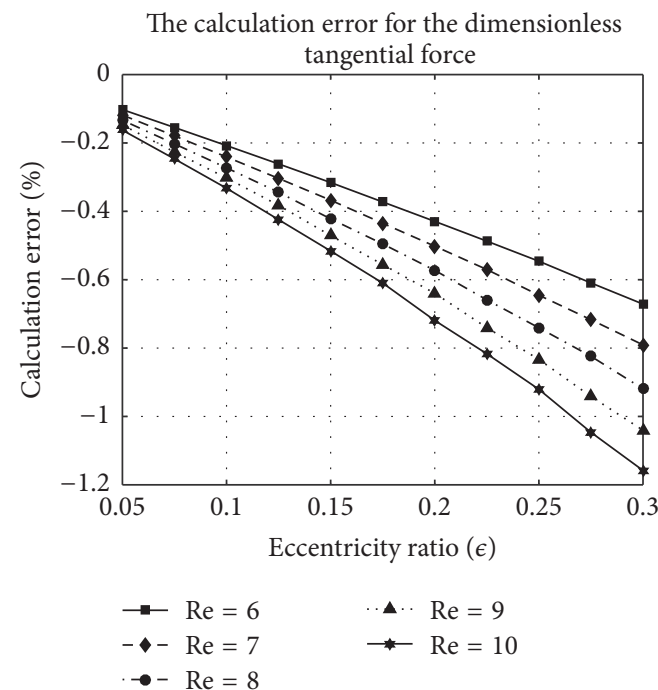

(d)

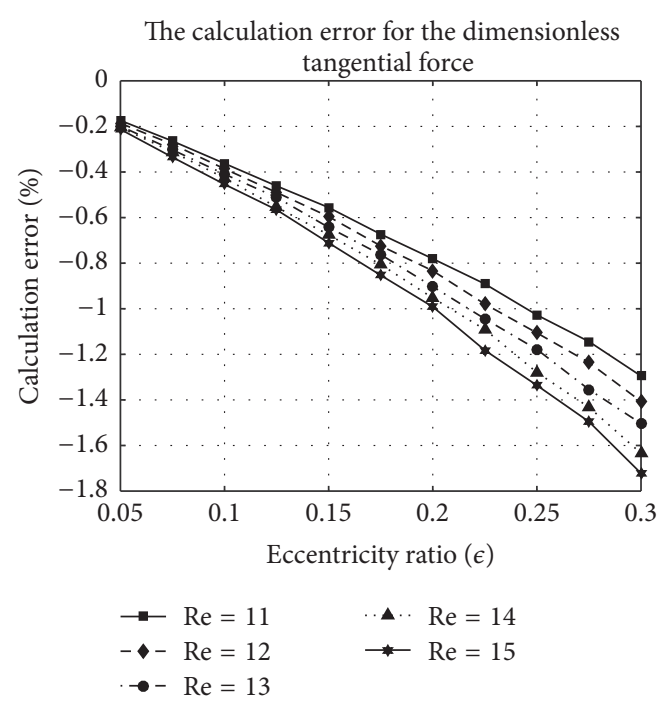

(f)

FIGURE 8: Contribution of the third-order nonlinear term in the pressure model to the dimensionless force components. 
bulk-flow technique. Finally, the lubricant pressure field is numerically integrated over the journal surface to determine the fluid film reaction forces.

The analysis of the pressure model confirms the significant contribution of the fluid inertia to the dynamics of the lubricant. According to the results, we have the following.

(1) Including the inertia effects increases the pressure magnitude, shifts the position of the pressure peak in the direction of the journal precession, and changes the shape of the pressure profile. The shape of the pressure is sinusoidal at small Reynolds numbers and it changes to cosine-like at high Reynolds numbers.

(2) The amplitudes of the pressure profile significantly change with eccentricity ratio.

(3) The slenderness ratio only influences the magnitude of the pressure profile.

(4) The direction and the magnitude of the radial forces change with inertia effects.

(5) The tangential forces are not significantly influenced by fluid inertia effects at small eccentricity ratios.

The results of this work are especially valuable to the highspeed turbomachinery industry (i.e., jet engines and gas turbines) since it provides a method to quickly and accurately review damper designs and provides results as inputs to the system engineering team during the conceptual design phase of an engine/turbine. This could lead to significant development cost reduction by decreasing the number of system design iterations. Furthermore, the damper model is effectively integrated into the rotordynamic model, providing a very powerful simulation tool to accurately predict the system vibrations during the development phase.

\section{Nomenclature}

$\begin{array}{ll}c: & \text { Radial clearance }(\mathrm{m}) \\ C_{f}: & \text { Force conversion coefficient } \\ C_{i j}: & \text { SFD damping coefficient }(\mathrm{N} \cdot \mathrm{s} / \mathrm{m}) \\ e: & \text { Eccentricity }(\mathrm{m}) \\ F_{r}, F_{t}: & \text { Radial and tangential fluid film } \\ h=c+e \cos \theta: & \text { reaction forces }(\mathrm{N}) \\ H=1+\varepsilon \cos \theta: & \text { Dimensionless fluid film thickness } \\ L: & \text { Journal length (m) } \\ M_{i j}: & \text { SFD inertia coefficient }(\mathrm{kg}) \\ P: & \text { Fluid pressure (Pa) } \\ R: & \text { Journal radius (m) } \\ \operatorname{Re}=\rho \omega c^{2} / \mu: & \text { Squeeze Reynolds number } \\ t: & \text { Time (s) } \\ u, v, w: & \text { Fluid velocity components in the } x, \\ \bar{F}=F c^{2} / R^{4} \omega \mu: & \text { Dimensionless fluid force } \\ \bar{P}=P c^{2} / R^{2} \omega \mu: & \text { Dimensionless fluid pressure } \\ \overline{P_{0}}: & \text { Dimensionless zeroth-order } \\ \bar{u}=u / R \omega: & \text { pressure } \\ & \text { Dimensionless circumferential } \\ & \text { velocity component }\end{array}$

$\begin{array}{ll}\bar{v}=v / c \omega: & \begin{array}{l}\text { Dimensionless radial velocity } \\ \text { component }\end{array} \\ \bar{w}=w / R \omega: & \begin{array}{l}\text { Dimensionless axial velocity } \\ \text { component }\end{array} \\ \varepsilon=e / c: & \text { Eccentricity ratio } \\ \eta=y / c: & \text { Dimensionless radial component } \\ \theta=\theta^{\prime}-\phi=x / R-\omega t: & \text { Dimensionless circumferential } \\ & \text { component }(\mathrm{rad}) \\ \lambda: & \text { Relaxation parameter } \\ \mu: & \text { Fluid dynamic viscosity }\left(\mathrm{N} \cdot \mathrm{s} / \mathrm{m}^{2}\right) \\ \xi=z / R: & \text { Dimensionless axial component } \\ \rho: & \text { Fluid density }\left(\mathrm{kg} / \mathrm{m}^{3}\right) \\ \tau=\omega t: & \text { Dimensionless temporal } \\ \omega: & \text { component } \\ & \text { Whirl velocity }(\mathrm{rad} / \mathrm{s}) .\end{array}$

\section{Competing Interests}

The authors declare that they have no competing interests.

\section{Acknowledgments}

This work was supported by grants from Natural Science and Engineering Research Council (NSERC) and Pratt and Whitney Canada.

\section{References}

[1] A. Z. Szeri, Fluid Film Lubrication: Theory and Design, Cambridge University Press, Cambridge, UK, 2005.

[2] S. Cooper, Preliminary Investigation of Oil Films for the Control of Vibration, Institution of Mechanical Engineers, 1963.

[3] L. Della Pietra and G. Adiletta, "The squeeze film damper over four decades of investigations-part I: characteristics and operating features," Shock and Vibration Digest, vol. 34, no. 1, pp. 3-26, 2002.

[4] L. Della Pietra and G. Adiletta, "The squeeze film damper over four decades of investigations. Part II: rotordynamic analyses with rigid and flexible rotors," Shock and Vibration Digest, vol. 34, no. 2, pp. 97-126, 2002.

[5] D. M. Smith, "Journal bearing dynamic characteristics-effect of inertia of lubricant," Proceedings of the Institution of Mechanical Engineers, Conference Proceedings, vol. 179, no. 10, pp. 37-44, 1964.

[6] T. Qingchang, L. Wei, and Z. Jun, "Fluid forces in short squeezefilm damper bearings," Tribology International, vol. 30, no. 10, pp. 733-738, 1997.

[7] S. Dousti, J. Cao, A. Younan, P. Allaire, and T. Dimond, "Temporal and convective inertia effects in plain journal bearings with eccentricity, velocity and acceleration," Journal of Tribology, vol. 134, no. 3, Article ID 031704, 2012.

[8] A. El-Shafei and S. Crandall, "Fluid inertia forces in squeeze film dampers," Rotating Machinery and Vehicle Dynamics, vol. 35, pp. 219-228, 1991.

[9] L. A. San Andres and J. M. Vance, "Effect of fluid inertia on squeeze-film damper forces for small-amplitude circular-centered motions," ASLE Transactions, vol. 30, no. 1, pp. 63-68, 1987.

[10] L. A. San Andrés, "Effect of fluid inertia on force coefficients for the long squeeze film damper," Tribology Transactions, vol. 31, no. 3, pp. 370-375, 1988. 
[11] L. A. San Andres and J. M. Vance, "Force coefficients for open-ended squeeze-film dampers executing small-amplitude motions about an off-center equilibrium position," ASLE Transactions, vol. 30, no. 1, pp. 69-76, 1987.

[12] L. A. San Andres and J. M. Vance, "Effects of fluid inertia on finite-length squeeze-film dampers," ASLE Transactions, vol. 30, no. 3, pp. 384-393, 1987.

[13] H. Hashimoto, "Boundary conditions for the calculation of the dynamic characteristics of infinitely long journal bearings with turbulence and inertia effects," Wear, vol. 96, no. 1, pp. 1-16, 1984.

[14] A. El-Shafei, "Unbalance response of a Jeffcott Rotor incorporating short squeeze film dampers," Journal of Engineering for Gas Turbines and Power, vol. 112, no. 4, pp. 445-453, 1990.

[15] A. El-Shafei, "Modeling fluid inertia forces of short journal bearings for rotordynamic applications," Journal of Vibration and Acoustics, vol. 117, no. 4, pp. 462-469, 1995.

[16] A. El-Shafei, "Unbalance response of a Jeffcott Rotor incorporating long squeeze film dampers," Journal of Vibration and Acoustics, vol. 113, no. 1, pp. 85-94, 1991.

[17] E. Reinhardt and J. W. Lund, "The influence of fluid inertia on the dynamic properties of journal bearings," Journal of Lubrication Technology, vol. 97, no. 2, pp. 159-167, 1975.

[18] W. Duan, F. Chu, C.-H. Kim, and Y.-B. Lee, "A bulk-flow analysis of static and dynamic characteristics of floating ring seals," Tribology International, vol. 40, no. 3, pp. 470-478, 2007.

[19] J. Gehannin, M. Arghir, and O. Bonneau, "Complete squeezefilm damper analysis based on the bulk flow equations," Tribology Transactions, vol. 53, no. 1, pp. 84-96, 2009.

[20] L. S. Andrés and A. Delgado, "A novel bulk-flow model for improved predictions of force coefficients in grooved oil seals operating eccentrically," Journal of Engineering for Gas Turbines and Power, vol. 134, no. 5, Article ID 052509, 2012.

[21] L. San Andrés and J. M. Vance, "Effects of fluid inertia and turbulence on the force coefficients for squeeze film dampers," Journal of Engineering for Gas Turbines and Power, vol. 108, no. 2, pp. 332-339, 1986.

[22] J. A. Tichy, "Effects of fluid inertia and viscoelasticity on the one-dimensional squeeze-film bearing," ASLE Transactions, vol. 27, no. 2, pp. 164-167, 1984.

[23] M. F. Modest and J. A. Tichy, "Squeeze film flow in arbitrarily shaped journal bearings subject to oscillations," Journal of Lubrication Technology, vol. 100, no. 2, pp. 323-329, 1978.

[24] C. H. T. Pan, "Calculation of pressure, shear, and flow in lubricating films for high speed bearings," Journal of Lubrication Technology, vol. 96, no. 1, pp. 80-94, 1974.

[25] J. M. Vance, Rotordynamics of Turbomachinery, vol. 9, John Wiley \& Sons, New York, NY, USA, 1988. 


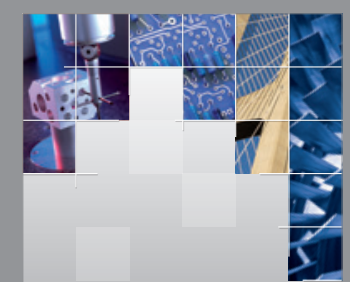

\section{Enfincering}
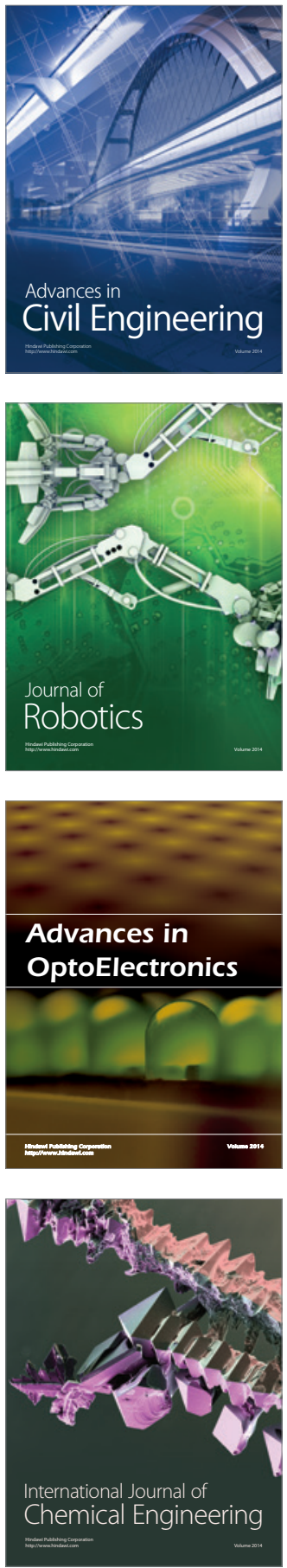

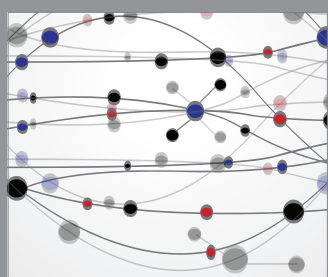

The Scientific World Journal

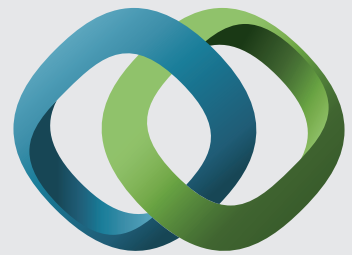

\section{Hindawi}

Submit your manuscripts at

https://www.hindawi.com
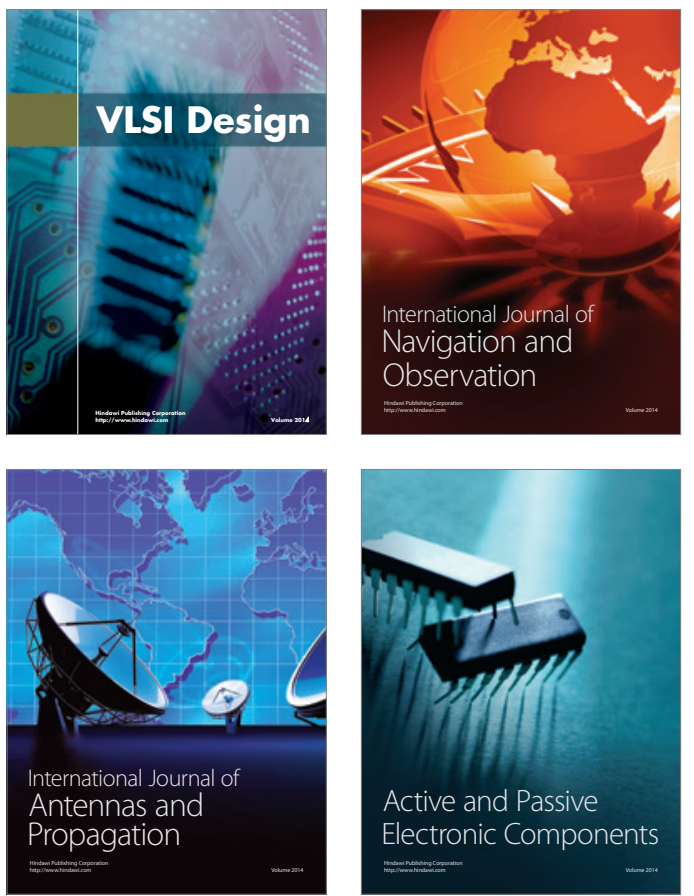
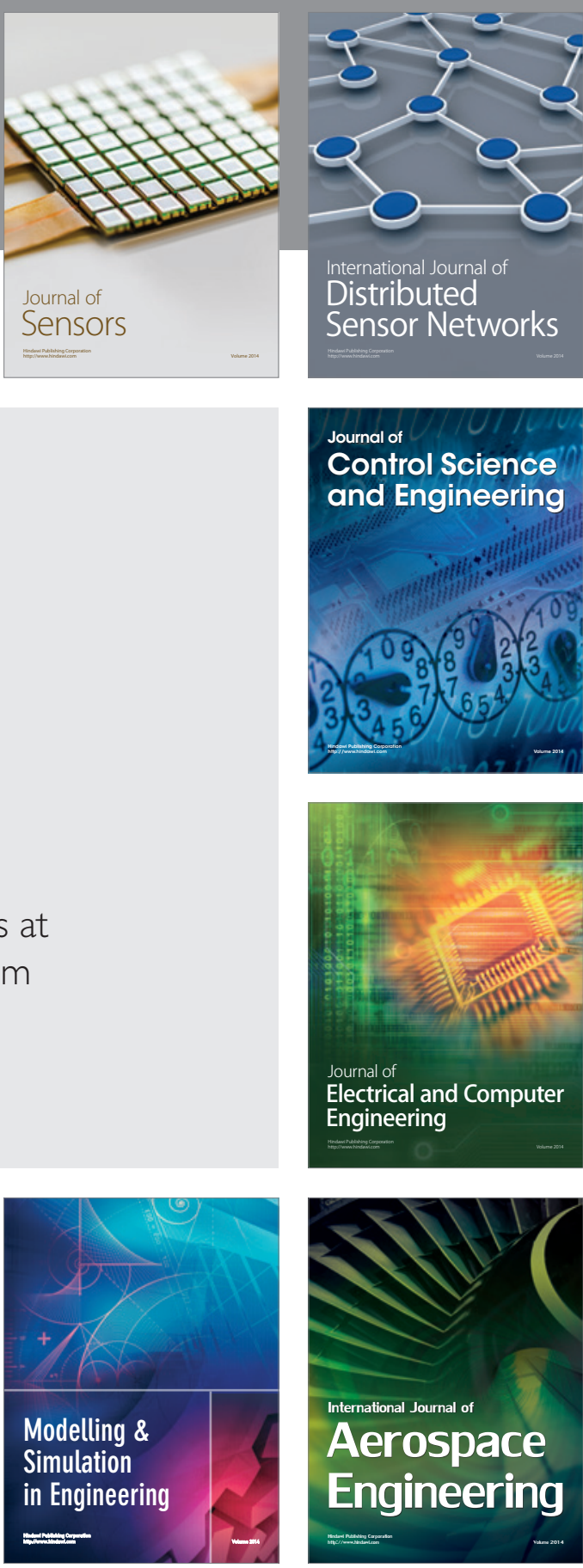

International Journal of

Distributed

Sensor Networks

$-$

Joumal of

Control Science

and Engineering
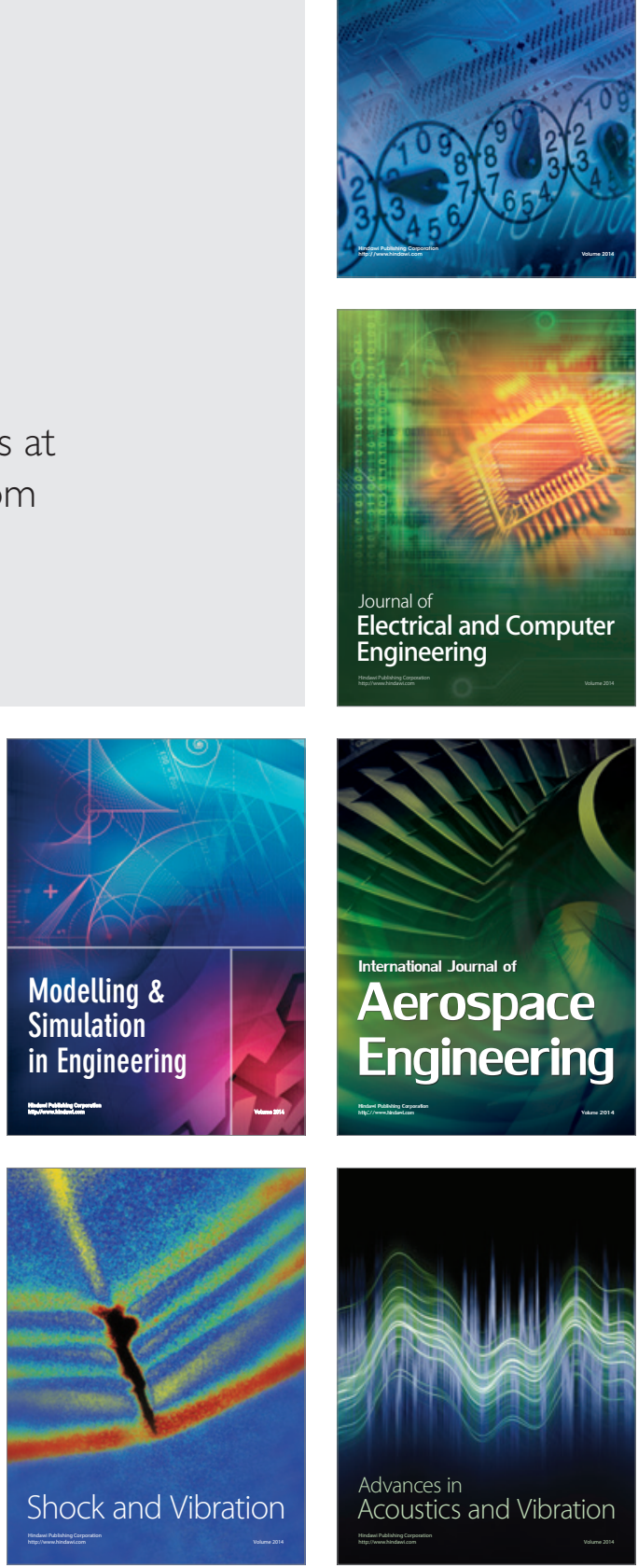